\title{
Orbit closures and rank schemes
}

Christine Riedtmann and Grzegorz Zwara

Dedicated to Andrzej Skowroński on the occasion of his 60th birthday

\begin{abstract}
Let $A$ be a finitely generated associative algebra over an algebraically closed field $k$, and consider the variety $\bmod _{A}^{d}(k)$ of $A$-module structures on $k^{d}$. In case $A$ is of finite representation type, equations defining the closure $\overline{\mathcal{O}}_{M}$ are known for $M \in \bmod _{A}^{d}(k)$; they are given by rank conditions on suitable matrices associated with $M$. We study the schemes $\varphi_{M}$ defined by such rank conditions for modules over arbitrary $A$, comparing them with similar schemes defined for representations of quivers and obtaining results on singularities. One of our main theorems is a description of the ideal of $\overline{\mathcal{O}}_{M}$ for a representation $M$ of a quiver of type $\mathbb{A}_{n}$, a result Lakshmibai and Magyar established for the equioriented quiver of type $\mathbb{A}_{n}$ in [12].
\end{abstract}

Mathematics Subject Classification (2010). Primary 14L30; Secondary 14B05, 16G20, $16 \mathrm{G} 70$.

Keywords. Modules, representations of quivers, orbit closures, subschemes, types of singularities.

\section{Introduction}

Throughout the paper, $k$ denotes an algebraically closed field of arbitrary characteristic. By abuse of notation, a $k$-scheme $\mathcal{X}$ and its functor of points, i.e. the functor from the category of commutative $k$-algebras to the category of sets sending $\mathcal{X}$ to the set of morphisms $\operatorname{Spec}(R) \rightarrow \mathcal{X}$, will be denoted by the same symbol. Any scheme $\mathcal{X}$ considered in the paper will be of finite type over $k$. In fact, $\mathcal{X}(k)$ can be viewed as the set of closed points of the scheme $\mathcal{X}$.

Let $d \in \mathbb{N}$. We denote by $\mathbb{M}_{d}$ the $k$-scheme of $d \times d$-matrices and by $\mathrm{GL}_{d}$ the group $k$-scheme of invertible $d \times d$-matrices. Let $A$ be a finitely generated associative $k$-algebra with a unit. The module scheme $\bmod _{A}^{d}$ can be easily described in terms of its functor of points

$$
\bmod _{A}^{d}(R)=\operatorname{Hom}_{\mathrm{k} \text {-alg. }}\left(A, \mathbb{M}_{d}(R)\right)
$$


The name is justified by the fact that $\bmod _{A}^{d}(k)$ can be identified with the set of $A$ module structures on the vector space $k^{d}$. The scheme $\bmod _{A}^{d}$ is affine and of finite type over $k$, so its coordinate ring $k\left[\bmod _{A}^{d}\right]$ is a finitely generated (commutative) $k$-algebra. The group scheme $\mathrm{GL}_{d}$ acts on $\bmod _{A}^{d}$ via

$$
(g \star M)(a)=g \cdot M(a) \cdot g^{-1} .
$$

Given $M \in \bmod _{A}^{d}(k)$, we denote its $\mathrm{GL}_{d}(k)$-orbit by $\mathcal{O}_{M}$. If we view the points of $\bmod _{A}^{d}(k)$ as $d$-dimensional $A$-modules, then $\mathcal{O}_{M}$ consists of all modules in $\bmod _{A}^{d}(k)$ isomorphic to $M$. By abuse of notation, we treat $\mathcal{O}_{M}$ and its closure $\overline{\mathcal{O}}_{M}$ as reduced subschemes of $\bmod _{A}^{d}$.

It is an open problem to describe the ideal of $\overline{\mathcal{O}}_{M}$ or even to exhibit polynomials having $\overline{\mathcal{O}}_{M}$ as their zero set. We now present some polynomials vanishing on $\overline{\mathcal{O}}_{M}$. Given $N \in \bmod _{A}^{d}$ and a $p \times q$-matrix $\underline{a}=\left(a_{i, j}\right)$ with coefficients in $A$ we define the $p d \times q d$-matrix

$$
N(\underline{a})=\left(\begin{array}{lll}
N\left(a_{1,1}\right) & \cdots & N\left(a_{1, q}\right) \\
\ldots \ldots \ldots & \ldots & \ldots \ldots . . . \\
N\left(a_{p, 1}\right) & \cdots & N\left(a_{p, q}\right)
\end{array}\right),
$$

and then any point $N \in \overline{\mathcal{O}}_{M}$ satisfies the condition

$$
\operatorname{rk} N(\underline{a}) \leq \operatorname{rk} M(\underline{a}),
$$

which means that all minors of size $1+\operatorname{rk} M(\underline{a})$ of the matrix $N(\underline{a})$ vanish. These minors can be interpreted as elements of the coordinate algebra $k\left[\bmod _{A}^{d}\right]$ (see Section 3 for details). Let $\mathcal{I}_{M}$ be the ideal in $k\left[\bmod _{A}^{d}\right]$ generated by such minors, where $\underline{a}$ varies over the set of all matrices with coefficients in $A$. Then $\mathcal{C}_{M}=\operatorname{Spec}\left(k\left[\bmod _{A}^{\bar{d}}\right] / \mathcal{I}_{M}\right)$ is a closed $\mathrm{GL}_{d}$-subscheme of $\bmod _{A}^{d}$ containing $\overline{\mathcal{O}}_{M}$.

When $A$ is a finite dimensional algebra, these rank conditions are directly related to the so-called Hom-order considered extensively before, for instance in [4], [5], [13], [15]. In fact, if $M, N \in \bmod _{A}^{d}(k), M \leq_{\text {hom }} N$ if and only if $N \in \mathcal{C}_{M}(k)$. It is known that $\left(\mathscr{C}_{M}\right)_{\text {red }}=\overline{\mathcal{O}}_{M}$ in special cases, e.g. if $A$ is a representation-finite algebra [15] or a tame concealed algebra [4]. However, $\left(\mathcal{C}_{M}\right)_{\text {red }}$ strictly contains $\overline{\mathcal{O}}_{M}$ in general; the first example is due to Carlson [13]. Moreover, $\mathcal{C}_{M}$ need not be reduced even if $\left(\mathcal{C}_{M}\right)_{\text {red }}=\overline{\mathcal{O}}_{M}$. This occurs already for the algebra $A=k[x] /\left(x^{2}\right)$ of dual numbers and dimension $d=2$ (see Example 3.7 for details).

Our goal in this article is to study the scheme $\mathcal{C}_{M}$ in its own right. We now roughly describe the content of every section.

In Section 2 we define rank ideals and present tools used later. The definition of the scheme $\mathcal{C}_{M}$ is given in Section 3, along with a reduction of the set of matrices $\underline{a}$ to be considered. In fact, a $p \times q$-matrix $\underline{a}$ with coefficients in $A$ yields a morphism $v_{\underline{a}}: A^{p} \rightarrow A^{q}$, and two matrices $\underline{a}$ and $\underline{a^{\prime}}$ yield the same rank conditions if $\underline{v}_{\underline{a}}$ and 
$v_{a^{\prime}}$ have isomorphic cokernels. In Section 4, we define analogous rank schemes for quiver representations and use them in Section 5 to extend Bongartz' results on a geometric version of the Morita equivalence to rank schemes.

In [12], Lakshmibai and Magyar proved a result which turns out to be equivalent to the following (see Section 4): If $M$ is a representation of an equioriented Dynkin quiver of type $\mathbb{A}$, then $\mathcal{C}_{M}=\left(\mathcal{C}_{M}\right)_{\text {red }}=\overline{\mathcal{O}}_{M}$. In [17], the second author introduced so-called hom-controlled exact functors. This tool allowed him to show that some types of singularities in orbit closures of modules over two different algebras coincide. In Section 6 we study hom-controlled exact functors for rank schemes, and we obtain one of our main results, a generalization of the result above to representations of Dynkin quivers of type $\mathbb{A}$, not necessarily equioriented (see Theorem 6.4). Thus the ideal defining $\overline{\mathcal{O}}_{M}$ is now known for Dynkin quivers of type $\mathbb{A}$; it is an open question whether this result can be generalized to representations of arbitrary Dynkin quivers.

The main advantage of the scheme $\mathcal{C}_{M}$ over $\overline{\mathcal{O}}_{M}$ is that its tangent space at some $N \in \overline{\mathcal{O}}_{M}$ has a module theoretic interpretation; we will explain this in Section 7 and use it in Section 8 to study the regularity of $\mathcal{C}_{M}$ at $N$. Under the assumption that the algebra is representation-finite, we will characterize the singular locus of $\mathcal{C}_{M}$. The motivation is that the knowledge of the singular locus for $\mathcal{C}_{M}$ helps to describe the singular locus for the orbit closure $\overline{\mathcal{O}}_{M}$. We will show in a forthcoming paper that in fact both loci coincide if $M$ is a nilpotent representation of an oriented cycle.

Acknowledgements. We would like to thank the Swiss Science Foundation for its support. This article was written while the second author was visiting the University of Berne; he also gratefully acknowledges support from the Research Grant No. N N201 269135 of the Polish Ministry of Science and High Education.

\section{Rank ideals}

Throughout the section $R$ denotes a commutative ring. Let $\mathbb{M}_{p \times q}$ be the affine scheme of $p \times q$-matrices, and fix $U \in \mathbb{M}_{p \times q}(R)$ and $t \in\{1, \ldots, \min (p, q)\}$. Following [7], 1.B, we denote by $I_{t}(U)$ the ideal in $R$ generated by the minors of $U$ of size $t$. It will be convenient to define $I_{0}(U)=R$ and $I_{t}(U)=0$ for $t>\min (p, q)$. Thus we have

$$
R=I_{0}(U) \supseteq I_{1}(U) \supseteq I_{2}(U) \supseteq \cdots .
$$

We first collect a few properties of $I_{t}(U)$.

Lemma 2.1. Let $U \in \mathbb{M}_{p \times q}(R)$ and $V \in \mathbb{M}_{q \times r}(R)$. Then

$$
I_{t}(U V) \subseteq I_{t}(U) \cap I_{t}(V) .
$$

Proof. Recall that, given a matrix $W \in \mathbb{M}_{p^{\prime} \times q^{\prime}}(R)$ and the corresponding $R$-homomorphism $U: R^{q^{\prime}} \rightarrow R^{p^{\prime}}$, the entries of the matrix of the $R$-homomorphism 
$\Lambda^{t}(U): \Lambda^{t}\left(R^{q^{\prime}}\right) \rightarrow \Lambda^{t}\left(R^{p^{\prime}}\right)$ with respect to the standard bases are just the $t \times t$ minors of $W$. For two subsets $K \subseteq\left\{1, \ldots, p^{\prime}\right\}, L \subseteq\left\{1, \ldots, q^{\prime}\right\}$ of the same cardinality, we denote the minor of $W$ corresponding to rows in $K$ and columns in $L$ by $W_{K, L}$. Using the functoriality of $\Lambda^{t}$, we obtain

$$
\operatorname{det}(U V)_{K, N}=\sum_{L} \operatorname{det} U_{K, L} \operatorname{det} V_{L, N}
$$

for any two subsets $K \subseteq\{1, \ldots, p\}, N \subseteq\{1, \ldots, r\}$ of cardinality $t$, where $L$ ranges over all subsets of $\{1, \ldots, q\}$ of cardinality $t$. Our claim follows.

Lemma 2.2. Let $U, V$ and $W$ be matrices with coefficients in $R$, where $U$ and $W$ are invertible and of a size that the product $U V W$ exists. Then $I_{t}(U V W)=I_{t}(V)$.

Proof. Apply Lemma 2.1 to $V^{\prime}=U V W$ and to $V=U^{-1} V^{\prime} W^{-1}$.

We leave the proof of the next lemma to the reader. Given two matrices $U$ and $V$ we set $U \oplus V=\left(\begin{array}{cc}U & 0 \\ 0 & V\end{array}\right)$.

Lemma 2.3. We have $I_{t}(U \oplus V)=\sum_{i=0}^{t} I_{i}(U) I_{t-i}(V)$. In particular, if $V$ is the identity matrix of size $s \leq t$, then $I_{t}(U \oplus V)=I_{t-s}(U)$.

\section{Definition and first properties of $\boldsymbol{C}_{M}$}

Let $A$ be a finitely generated associative $k$-algebra and $d \in \mathbb{N}$. The coordinate algebra $k\left[\bmod _{A}^{d}\right]$ can be constructed as follows: Choosing generators $a_{1}, \ldots, a_{r}$ of $A$ we obtain an isomorphism of $A$ with the quotient of a free $k$-algebra $k\left\langle x_{1}, \ldots, x_{r}\right\rangle$ by a two-sided ideal $J$. We consider $r d^{2}$ independent variables $x_{i, j}^{l}, l \leq r, i, j \leq d$, arranged into $r$ matrices $X_{l}=\left(x_{i, j}^{l}\right)$ of size $d \times d$. Then $k\left[\bmod _{A}^{d}\right]$ is the quotient of the polynomial algebra $k\left[x_{i, j}^{l}\right]$ by the ideal generated by the entries of the $d \times d$ matrices $\rho\left(X_{1}, \ldots, X_{r}\right), \rho \in J$. Let $X_{A}^{d}$ be the element in $\bmod _{A}^{d}\left(k\left[\bmod _{A}^{d}\right]\right)$ defined by the equalities $X\left(a_{l}\right)=\bar{X}_{l}$ for $l \leq r$. We call $X_{A}^{d}$ a universal module in $\bmod _{A}^{d}$, as it satisfies the following universal property: For any commutative $k$-algebra $R$ and any element $N \in \bmod _{A}^{d}(R)$ there is a unique algebra homomorphism $\varphi: k\left[\bmod _{A}^{d}\right] \rightarrow R$ such that $N=\bmod _{A}^{d}(\varphi)\left(X_{A}^{d}\right)$.

The coordinate algebra $k\left[\mathbb{M}_{p \times q}\right]$ is the polynomial algebra $k\left[y_{i, j}\right]$ with $i \leq p$, $j \leq q$. We denote by $\mathcal{V}_{p \times q}^{r} \subseteq \mathbb{M}_{p \times q}$ the closed subscheme of "matrices of rank at most $r$ " defined by the ideal $I_{r+1}(Y) \subseteq k\left[\mathbb{M}_{p \times q}\right]$ for $Y=\left(y_{i, j}\right) \in \mathbb{M}_{p \times q}\left(k\left[\mathbb{M}_{p \times q}\right]\right)$.

Let $\underline{a}=\left(a_{i, j}\right)$ be a $p \times q$ matrix with coefficients in $A$. The assignment

$$
N \mapsto N(\underline{a})=\left(\begin{array}{lll}
N\left(a_{1,1}\right) & \ldots & N\left(a_{1, q}\right) \\
\ldots \ldots \ldots \ldots & \ldots \ldots \ldots \\
N\left(a_{p, 1}\right) & \cdots & N\left(a_{p, q}\right)
\end{array}\right)
$$


leads to a regular morphism $\Theta_{\underline{a}}: \bmod _{A}^{d} \rightarrow \mathbb{M}_{p d \times q d}$. For an $A$-module $M \in$ $\bmod _{A}^{d}(k)$, we set

$$
\mathcal{C}_{M, \underline{a}}=\Theta_{\underline{a}}^{-1}\left(\mathcal{V}_{p d \times q d}^{\mathrm{rk} M(a)}\right) .
$$

Note that $\mathscr{C}_{M, \underline{a}}=\operatorname{Spec}\left(k\left[\bmod _{A}^{d}\right] / \mathcal{I}_{M, \underline{a}}\right)$, where $\mathcal{I}_{M, \underline{a}}=I_{1+\mathrm{rk} M(\underline{a})}\left(X_{A}^{d}(\underline{a})\right) \subseteq$ $k\left[\bmod _{A}^{d}\right]$.

Lemma 3.1. The subscheme $\mathcal{C}_{M, \underline{a}} \subseteq \bmod _{A}^{d}$ is stable under $\mathrm{GL}_{d}$.

Proof. Fix a commutative $k$-algebra $R$. We need to show that $N \in \mathcal{C}_{M, a}(R)$ and $g \in \mathrm{GL}_{d}(R)$ implies $g * N \in \mathcal{C}_{M, a}(R)$, or equivalently that all $r \times r$-minors of $(g * N)(\underline{a})$ vanish, where $r=1+\operatorname{rk} M(\underline{a})$. But $I_{r}(g * N(\underline{a}))=I_{r}(N(\underline{a}))$ by Lemma 2.2 as

$$
(g * N)(\underline{a})=\left(\begin{array}{cccc}
g & 0 & \cdots & 0 \\
0 & g & \cdots & 0 \\
\ldots & \ldots & \ldots & \ldots \\
0 & 0 & \cdots & g
\end{array}\right) \cdot N(\underline{a}) \cdot\left(\begin{array}{cccc}
g^{-1} & 0 & \cdots & 0 \\
0 & g^{-1} & \cdots & 0 \\
\ldots \ldots & \ldots & \ldots & \ldots \\
0 & 0 & \cdots & g^{-1}
\end{array}\right) .
$$

We define the rank scheme associated to $M$ as

$$
e_{M}=\bigcap \varphi_{M, \underline{a}},
$$

where $\underline{a}$ ranges over all $p \times q$-matrices with coefficients in $A$ for all $p$ and $q$. Thus $\mathcal{C}_{M}$ is the closed $\mathrm{GL}_{d}$-subscheme of $\bmod _{A}^{d}$ defined by $\mathcal{I}_{M}=\sum \mathcal{I}_{M, \underline{a}}$. Note that isomorphic modules define the same rank scheme.

Sending $a \in A$ to the $A$-homomorphism $v_{a}: A \rightarrow A, v_{a}(b)=b \cdot a$ defines a bijection from $A$ to $\operatorname{Hom}_{A}(A, A)$. Given a $p \times q$-matrix $\underline{a}=\left(a_{i, j}\right)$ with coefficients in $A$, we define an $A$-homomorphism

$$
v_{\underline{a}}: A^{p} \stackrel{\left(v_{a_{j, i}}\right)}{\longrightarrow} A^{q} .
$$

This gives a bijection between the set of $p \times q$-matrices with coefficients in $A$ and the space $\operatorname{Hom}_{A}\left(A^{p}, A^{q}\right)$. Moreover, for a $q \times s$-matrix $\underline{b}$ with coefficients in $A$ we get

$$
v_{\underline{a} \cdot \underline{b}}=v_{\underline{b}} \circ v_{\underline{a}} .
$$

Lemma 3.2. Let $\underline{a}^{\prime}$ and $\underline{a}^{\prime \prime}$ be two matrices with coefficients in A. If the cokernels $\operatorname{Coker}\left(v_{\underline{a}^{\prime}}\right)$ and $\operatorname{Coker}\left(v_{\underline{a}^{\prime \prime}}\right)$ are A-isomorphic then $\mathcal{I}_{M, \underline{a}^{\prime}}=\mathcal{I}_{M, \underline{a}^{\prime \prime}}$.

As a consequence, we obtain a well defined scheme $\boldsymbol{C}_{M, L}$ for any finitely presented $A$-module $L$ by choosing a presentation

$$
A^{p} \stackrel{\nu_{a}}{\rightarrow} A^{q} \rightarrow L \rightarrow 0
$$


and setting $\boldsymbol{C}_{M, L}=\boldsymbol{C}_{M, \underline{a}}$. Note that $\boldsymbol{\ell}_{M}=\bigcap \boldsymbol{C}_{M, L}$, where $L$ ranges over representatives of all isomorphism classes of finitely presented $A$-modules.

Proof of the lemma. Let $\underline{a}^{\prime}$ and $\underline{a}^{\prime \prime}$ be two matrices with coefficients in $A$ of sizes $p_{1} \times q_{1}$ and $p_{2} \times q_{2}$, respectively. Setting $f_{1}=v_{\underline{a}^{\prime}}$ and $f_{2}=v_{a^{\prime \prime}}$ we obtain two $A$-homomorphisms

$$
A^{p_{1}} \stackrel{f_{1}}{\rightarrow} A^{q_{1}} \quad \text { and } \quad A^{p_{2}} \stackrel{f_{2}}{\longrightarrow} A^{q_{2}} .
$$

We assume that there is an $A$-isomorphism $\xi: \operatorname{Coker}\left(f_{1}\right) \rightarrow \operatorname{Coker}\left(f_{2}\right)$. We claim that there are matrices $\underline{b}$ and $\underline{c}$ with coefficients in $A$ such that

$$
\left(\begin{array}{cc}
\underline{a}^{\prime} & 0 \\
0 & 1_{d q_{2}}
\end{array}\right)=\underline{b} \cdot\left(\begin{array}{cc}
1_{d q_{1}} & 0 \\
0 & \underline{a}^{\prime \prime}
\end{array}\right) \cdot \underline{c} .
$$

Using the property that free $A$-modules are projective we obtain the following commutative diagram with exact rows

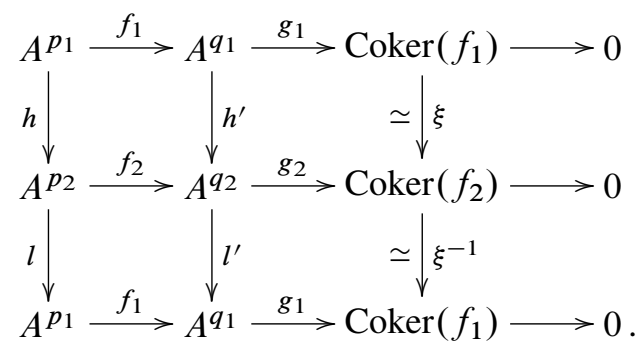

In particular,

$$
h^{\prime} f_{1}=f_{2} h, \quad l^{\prime} f_{2}=f_{1} l,
$$

and $g_{1} l^{\prime} h^{\prime}=g_{1}$. The latter implies that $\operatorname{Im}\left(1-l^{\prime} h^{\prime}\right)$ is contained in $\operatorname{Im}\left(f_{1}\right)$, and consequently $1-l^{\prime} h^{\prime}$ factors through $f_{1}$. From this, and by symmetry, we get two $A$-homomorphisms $\varphi_{i}: A^{q_{i}} \rightarrow A^{p_{i}}, i=1,2$, such that

$$
l^{\prime} h^{\prime}+f_{1} \varphi_{1}=1_{A^{q_{1}}} \quad \text { and } \quad h^{\prime} l^{\prime}+f_{2} \varphi_{2}=1_{A^{q_{2}}} .
$$

We conclude from (3.1) and (3.2) that

$$
\left(\begin{array}{cc}
f_{1} & 0 \\
0 & 1_{A^{q_{2}}}
\end{array}\right)=\left(\begin{array}{cc}
f_{1} \varphi_{1} & -l^{\prime} \\
h^{\prime} & 1_{A^{q_{2}}}
\end{array}\right) \cdot\left(\begin{array}{cc}
1_{A^{q_{1}}} & 0 \\
0 & f_{2}
\end{array}\right) \cdot\left(\begin{array}{cc}
f_{1} & l^{\prime} \\
-h & \varphi_{2}
\end{array}\right) .
$$

We get the claim by choosing matrices $\underline{b}$ and $\underline{c}$ such that

$$
v_{\underline{b}}=\left(\begin{array}{cc}
f_{1} & l^{\prime} \\
-h & \varphi_{2}
\end{array}\right) \quad \text { and } \quad v_{\underline{c}}=\left(\begin{array}{cc}
f_{1} \varphi_{1} & -l^{\prime} \\
h^{\prime} & 1_{A^{q_{2}}}
\end{array}\right) .
$$


Let $X=X_{A}^{d}$ be a universal module for $\bmod _{A}^{d}$. The claim implies that

$$
\left(\begin{array}{cc}
X\left(\underline{a}^{\prime}\right) & 0 \\
0 & 1_{d q_{2}}
\end{array}\right)=X(\underline{b}) \cdot\left(\begin{array}{cc}
1_{d q_{1}} & 0 \\
0 & X\left(\underline{a}^{\prime \prime}\right)
\end{array}\right) \cdot X(\underline{c})
$$

By Lemmas 2.1 and 2.3, we know that

$$
I_{t-d q_{2}}\left(X\left(\underline{a}^{\prime}\right)\right)=I_{t}\left(\begin{array}{cc}
X\left(\underline{a}^{\prime}\right) & 0 \\
0 & 1_{d q_{2}}
\end{array}\right) \subseteq I_{t}\left(\begin{array}{cc}
1_{d q_{1}} & 0 \\
0 & X\left(\underline{a}^{\prime \prime}\right)
\end{array}\right)=I_{t-d q_{1}}\left(X\left(\underline{a}^{\prime \prime}\right)\right) .
$$

for any $t \geq q_{1} d, q_{2} d$. Applying the functor $\operatorname{Hom}_{A}(-, M)$ we obtain the exact sequences

$$
\begin{aligned}
& 0 \rightarrow \operatorname{Hom}_{A}\left(\operatorname{Coker}\left(f_{1}\right), M\right) \rightarrow \operatorname{Hom}_{A}\left(A^{q_{1}}, M\right) \stackrel{\operatorname{Hom}_{A}\left(v_{\underline{a}^{\prime}}, M\right)}{\longrightarrow} \operatorname{Hom}_{A}\left(A^{\left.p_{1}, M\right),}\right. \\
& 0 \rightarrow \operatorname{Hom}_{A}\left(\operatorname{Coker}\left(f_{2}\right), M\right) \rightarrow \operatorname{Hom}_{A}\left(A ^ { q _ { 2 } , M ) } \stackrel { \operatorname { H o m } _ { A } ( v _ { \underline { a } ^ { \prime \prime } } , M ) } { \longrightarrow } \operatorname { H o m } _ { A } \left(A^{\left.p_{2}, M\right) .}\right.\right.
\end{aligned}
$$

Let $w=\operatorname{dim}_{k} \operatorname{Hom}_{A}\left(\operatorname{Coker}\left(f_{1}\right), M\right)=\operatorname{dim}_{k} \operatorname{Hom}_{A}\left(\operatorname{Coker}\left(f_{2}\right), M\right)$. Identifying the space $\operatorname{Hom}_{A}\left(A^{s}, M\right)$ with $k^{d s}, s \in \mathbb{N}$, we get

$$
\operatorname{Hom}_{A}\left(v_{\underline{a}^{\prime}}, M\right)=M\left(\underline{a}^{\prime}\right) \text { and } \operatorname{Hom}_{A}\left(\underline{v}_{\underline{a}^{\prime \prime}}, M\right)=M\left(\underline{a}^{\prime \prime}\right)
$$

Consequently,

$$
\operatorname{rk}\left(M\left(\underline{a}^{\prime}\right)\right)=d q_{1}-w, \quad \operatorname{rk}\left(M\left(\underline{a}^{\prime \prime}\right)\right)=d q_{2}-w
$$

and

$$
\mathcal{I}_{M, \underline{a}^{\prime}}=I_{1+d q_{1}-w}\left(X\left(\underline{a}^{\prime}\right)\right) \subseteq I_{1+d q_{2}-w}\left(X\left(\underline{a}^{\prime \prime}\right)\right)=\mathcal{I}_{M, \underline{a}^{\prime \prime}}
$$

In a similar way we prove the reverse inclusion, which finishes the proof.

Lemma 3.3. For finitely presented A-modules $L_{1}$ and $L_{2}$, we have that $\mathcal{I}_{M, L_{1} \oplus L_{2} \subseteq} \subseteq$ $\mathcal{I}_{M, L_{1}}+\mathcal{I}_{M, L_{2}}$.

Proof. We fix matrices $\underline{a}^{\prime}$ and $\underline{a}^{\prime \prime}$ with coefficients in $A$ such that the cokernels of $v_{\underline{a}^{\prime}}$ and $v_{\underline{a}^{\prime \prime}}$ are isomorphic to $L_{1}$ and $L_{2}$, respectively. Let $r_{1}=\operatorname{rk}\left(M\left(\underline{a}^{\prime}\right)\right)$ and $r_{2}=\operatorname{rk}\left(M\left(\underline{a}^{\prime \prime}\right)\right)$, and set $X=X_{A}^{d}$. Using the fact that

$$
R=I_{0}(U) \supseteq I_{1}(U) \supseteq I_{2}(U) \supseteq \cdots
$$


for any matrix $U$ with coefficients in a commutative ring $R$, we get from Lemma 2.3

$$
\begin{aligned}
\mathcal{I}_{M, L_{1} \oplus L_{2}} & =\mathcal{I}_{M, \underline{a}^{\prime} \oplus \underline{a}^{\prime \prime}}=I_{1+r_{1}+r_{2}}\left(X\left(\underline{a}^{\prime}\right) \oplus X\left(\underline{a}^{\prime \prime}\right)\right) \\
& =\sum_{t=0}^{1+r_{1}+r_{2}} I_{t}\left(X\left(\underline{a}^{\prime}\right)\right) \cdot I_{1+r_{1}+r_{2}-t}\left(X\left(\underline{a}^{\prime \prime}\right)\right) \\
& \subseteq \sum_{t=0}^{r_{1}} I_{1+r_{1}+r_{2}-t}\left(X\left(\underline{a}^{\prime \prime}\right)\right)+\sum_{t=1+r_{1}}^{1+r_{1}+r_{2}} I_{t}\left(X\left(\underline{a}^{\prime}\right)\right) \\
& =I_{1+r_{2}}\left(X\left(\underline{a}^{\prime \prime}\right)\right)+I_{1+r_{1}}\left(X\left(\underline{a}^{\prime}\right)\right)=\mathcal{I}_{M, \underline{a}^{\prime \prime}}+\mathcal{I}_{M, \underline{a}^{\prime}} \\
& =\mathcal{I}_{M, L_{1}}+\mathfrak{I}_{M, L_{2}} .
\end{aligned}
$$

Let $L$ be a finitely presented $A$-module. Then the space $\operatorname{Hom}_{A}(L, M)$ is finite dimensional, and we choose a basis $f_{1}, \ldots, f_{s}$. We denote by $L_{M}$ the kernel of the map

$$
L \stackrel{\left(f_{1}, \ldots, f_{s}\right)^{t}}{\longrightarrow} M^{s} .
$$

Note that $L_{M}$ does not depend on the choice of the basis $f_{1}, \ldots, f_{s}$.

Lemma 3.4. Using the above notation, we have $\mathcal{I}_{M, L} \subseteq \mathcal{I}_{M, L / L_{M}}$.

Proof. As there is an injective $A$-homomorphism from $L / L_{M}$ to $M^{s}$, the module $L / L_{M}$ is finite dimensional and thus finitely presented, as $A$ is finitely generated. We may choose presentations of $L$ and of $L / L_{M}$ for which there is a commutative diagram

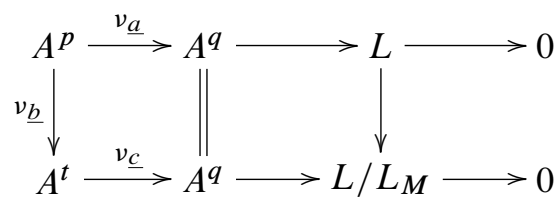

with exact rows.

From $v_{\underline{a}}=v_{\underline{c}} \circ v_{\underline{b}}$ we see that $\underline{a}=\underline{b} \circ \underline{c}$. Note that the injection from $L / L_{M}$ to $M^{s}$ induces an isomorphism from $\operatorname{Hom}_{A}\left(L / L_{M}, M\right)$ to $\operatorname{Hom}_{A}(L, M)$ and thus $\operatorname{rk}(M(\underline{a}))=\operatorname{rk}(M(\underline{c}))$. By Lemma 2.1 we conclude that

$$
\begin{aligned}
\mathcal{I}_{M, L} & =\mathcal{I}_{M, \underline{a}}=I_{1+\mathrm{rk} M(a)}\left(X_{A}^{d}(\underline{a})\right) \\
& =I_{1+\mathrm{rk} M(\underline{a})}\left(X_{A}^{d}(\underline{b}) \circ X_{A}^{d}(\underline{c})\right) \\
& \subseteq I_{1+\mathrm{rk} M(\underline{c})}\left(X_{A}^{d}(\underline{c})\right)=I_{M, \underline{c}} \\
& =I_{M, L / L_{M}} .
\end{aligned}
$$

Next we study the behavior of rank schemes under an algebra homomorphism $\varphi: A \rightarrow B$. For a $p \times q$-matrix $\underline{a}=\left(a_{i, j}\right)$ with coefficients in $A$, we denote 
the corresponding $p \times q$-matrix with coefficients in $B$ by $\varphi(\underline{a})=\left(\varphi\left(a_{i, j}\right)\right)$. Any $B$-module can be considered as an $A$-module via $\varphi$; we will write ${ }_{A} M$ for the $A$ module corresponding to the $B$-module ${ }_{B} M$. In addition, $\varphi$ induces a regular $\mathrm{GL}_{d^{-}}$ morphism $\varphi^{d}: \bmod _{B}^{d} \rightarrow \bmod _{A}^{d}$ defined by $\left[\varphi^{d}(N)\right](a)=N(\varphi(a))$, which is a closed immersion if $\varphi$ is surjective. If $d=\operatorname{dim}_{k} M$, then $\varphi^{d}\left(\mathcal{O}_{B} M\right)=\mathcal{O}_{A} M$, and consequently $\overline{\mathcal{O}}_{B} M \subseteq\left(\varphi^{d}\right)^{-1}\left(\overline{\mathcal{O}}_{A} M\right)$. A similar result holds for rank schemes.

Lemma 3.5. Let $\varphi: A \rightarrow B$ be an algebra homomorphism and let $M$ belong to $\bmod _{B}^{d}(k)$. Then

$$
\bigodot_{B} M \subseteq\left(\varphi^{d}\right)^{-1}\left(\bigodot_{A} M\right)
$$

If $\varphi$ is surjective, the above inclusion is an equality.

Proof. Note that $\Theta_{\varphi(a)}=\Theta_{\underline{a}} \circ \varphi^{d}$ and ${ }_{B} M(\varphi(\underline{a}))={ }_{A} M(\underline{a})$ for a $B$-module ${ }_{B} M$, and thus $\mathcal{C}_{B} M, \varphi(a)=\left(\varphi^{d}\right)^{-1}\left(\mathcal{C}_{A} M,(a)\right)$.

The algebra $B=A$ / Ann $M$ is finite dimensional, being a subalgebra of $\operatorname{End}_{k}(M)$. By the above lemma, we can work over the finite dimensional algebra $B=A$ / Ann $M$ instead of $A$ and consider $M$ as a $B$-module. For a finite dimensional algebra, any finitely presented module is isomorphic to a direct sum of indecomposables, and we obtain the following consequence.

Corollary 3.6. Let $A$ be finite dimensional, and let $\mathscr{L}$ be a complete set of pairwise non-isomorphic indecomposable A-modules which can be embedded into finite powers of $M$. Then

$$
\mathcal{I}_{M}=\sum_{L \in \mathscr{L}} \mathcal{I}_{M, L}
$$

We construct $\mathcal{C}_{M}$ on a simple but instructive example.

Example 3.7. Let $A=k[\varepsilon] \simeq k[x] /\left(x^{2}\right)$ be the algebra of dual numbers and $M: A \rightarrow \mathbb{M}_{2}(k)$ be the unique algebra homomorphism satisfying $M(\varepsilon)=\left[\begin{array}{ll}0 & 0 \\ 1 & 0\end{array}\right]$, so that the corresponding module is isomorphic to ${ }_{A} A$. Choosing $\varepsilon$ as a generator of $A$, we identify the coordinate algebra $k\left[\bmod _{A}^{2}\right]$ with

$$
k\left[x_{1,1}, x_{1,2}, x_{2,1}, x_{2,2}\right] /\left(\text { entries of }\left(\begin{array}{ll}
x_{1,1} & x_{1,2} \\
x_{2,1} & x_{2,2}
\end{array}\right)^{2}\right) .
$$

The set $\mathscr{L}$ considered in the previous corollary consists of two modules: $M$ and its one-dimensional simple submodule denoted by $S$. In fact, any indecomposable $A$-module is isomorphic to either $M$ or $S$, so the algebra $A$ is representation finite and therefore $\left(\mathcal{C}_{M}\right)_{\text {red }}=\overline{\mathcal{O}}_{M}$, as mentioned in the introduction. Since $M$ is free as an $A$-module, we have a free presentation $0=A^{0} \rightarrow A^{1} \rightarrow M \rightarrow 0$ giving us no condition, i.e. $\mathcal{I}_{M, M}=0$. Thus choosing a free presentation $A^{1} \stackrel{\nu_{(\varepsilon)}}{\longrightarrow} A^{1} \rightarrow S \rightarrow 0$ 
and denoting by $\bar{x}_{i, j}$ the residue class of $x_{i, j}$ in the coordinate algebra $k\left[\bmod _{A}^{2}\right]$, we get

$$
\mathcal{I}_{M}=\mathcal{I}_{M, S}=I_{1+\mathrm{rk} M(\varepsilon)}\left(\begin{array}{ll}
\bar{x}_{1,1} & \bar{x}_{1,2} \\
\bar{x}_{2,1} & \bar{x}_{2,2}
\end{array}\right)=I_{2}\left(\begin{array}{ll}
\bar{x}_{1,1} & \bar{x}_{1,2} \\
\bar{x}_{2,1} & \bar{x}_{2,2}
\end{array}\right)=\left(\operatorname{det}\left(\begin{array}{ll}
\bar{x}_{1,1} & \bar{x}_{1,2} \\
\bar{x}_{2,1} & \bar{x}_{2,2}
\end{array}\right)\right) .
$$

Obviously the trace of $\left(\begin{array}{ll}\bar{x}_{1,1} & \bar{x}_{1,2} \\ \bar{x}_{2,1} & \bar{x}_{2,2}\end{array}\right)$ does not belong to $\mathcal{I}_{M}$ (but its third power does), so the ideal $\mathcal{I}_{M}$ is not radical and $\mathscr{C}_{M}$ is not reduced.

\section{Rank schemes for representations of quivers}

We first recall the classical definition of the representation space of a quiver with relations for a given dimension vector, acted upon by a product of general linear groups, and then view this space as the $k$-points of an affine scheme with the action of a group scheme.

Let $Q=\left(Q_{0}, Q_{1}, s, t\right)$ be a finite quiver, i.e. a finite set $Q_{0}$ of vertices and a finite set $Q_{1}$ of arrows $\alpha: s \alpha \rightarrow t \alpha$, where $s \alpha$ and $t \alpha$ denote the starting and the terminating vertex of $\alpha$, respectively. A representation of $Q$ over $k$ is a collection $\left(X(i) ; i \in Q_{0}\right)$ of finite dimensional $k$-vector spaces together with a collection $\left(X(\alpha): X(s \alpha) \rightarrow X(t \alpha) ; \alpha \in Q_{1}\right)$ of $k$-linear maps. A morphism $f: X \rightarrow Y$ between two representations is a collection $(f(i): X(i) \rightarrow Y(i))$ of $k$-linear maps such that

$$
f(t \alpha) \circ X(\alpha)=Y(\alpha) \circ f(s \alpha) \quad \text { for all } \alpha \in Q_{1} .
$$

The dimension vector of a representation $X$ of $Q$ is the vector

$$
\operatorname{dim} X=(\operatorname{dim} X(i)) \in \mathbb{N}^{Q_{0}} .
$$

We denote the category of representations of $Q$ by $\operatorname{rep}(Q)$, and for any vector $\boldsymbol{d}=$ $\left(d_{i}\right) \in \mathbb{N}^{Q_{0}}$,

$$
\operatorname{rep}_{Q}^{\boldsymbol{d}}(k)=\prod_{\alpha \in Q_{1}} \mathbb{M}_{d_{t \alpha} \times d_{s \alpha}}(k)
$$

is the affine space of representations $X$ of $Q$ with $X(i)=k^{d_{i}}, i \in Q_{0}$. The group

$$
\mathrm{GL}_{\boldsymbol{d}}(k)=\prod_{i \in Q_{0}} \mathrm{GL}_{d_{i}}(k)
$$

acts on $\operatorname{rep}_{Q}^{\boldsymbol{d}}(k)$ by

$$
\left(\left(g_{i}\right) \star X\right)(\alpha)=g_{t \alpha} \circ X(\alpha) \circ g_{s \alpha}^{-1} .
$$

Note that the $\mathrm{GL}_{\boldsymbol{d}}(k)$-orbit of $X$, denoted by $\mathcal{O}_{X}$, consists of the representations $Y$ in $\operatorname{rep}_{Q}^{d}(k)$ which are isomorphic to $X$. 
Let $k Q$ denote the path algebra of $Q$ : The paths in $Q$ form a $k$-basis of $k Q$, and two paths are multiplied by juxtaposing them if possible and they have product 0 otherwise. In each vertex $i$ of $Q$ we have the trivial path $\varepsilon_{i}$ of length zero. Note that

$$
1_{k Q}=\sum_{i \in Q_{0}} \varepsilon_{i}
$$

is a decomposition of 1 into a sum of pairwise orthogonal idempotents and that $\varepsilon_{i} \cdot k Q \cdot \varepsilon_{j}$ is the vector subspace consisting of the linear combinations $\omega$ of paths starting from vertex $j$ and terminating at $i$. We will write $s(\omega)=j$ and $t(\omega)=i$. For any representation $X \in \operatorname{rep}_{Q}^{\boldsymbol{d}}(k)$ the $d_{i} \times d_{j}$-matrix $X(\omega)$ is defined in the obvious way. If $J$ is a two-sided ideal of $k Q$, one can restrict the category $\operatorname{rep}(Q)$ to the full subcategory $\operatorname{rep}(Q, J)$ consisting of the representations annihilated by $J$. The pair $(Q, J)$ is called a bound quiver if $J$ is an admissible ideal, i.e. $\left(k Q_{+}\right)^{N} \subseteq J \subseteq$ $\left(k Q_{+}\right)^{2}$ for some $N \geq 2$, where $k Q_{+}$stands for the ideal in $k Q$ spanned by the paths of positive length.

The affine scheme $\operatorname{rep}_{Q}^{d}$ is defined as

$$
\operatorname{rep}_{Q}^{d}=\prod_{\alpha \in Q_{1}} \mathbb{M}_{d_{t \alpha} \times d_{s \alpha}}
$$

and has the polynomial ring

$$
k\left[\operatorname{rep}_{Q}^{\boldsymbol{d}}\right]=k\left[x_{k l}^{\alpha}\right]
$$

as its coordinate ring, where $\alpha$ ranges over $Q_{1}, k$ over $\left\{1, \ldots, d_{t \alpha}\right\}$, and $l$ over $\left\{1, \ldots, d_{s \alpha}\right\}$. A universal representation $X_{Q}^{\boldsymbol{d}}$ is given by $X_{Q}^{\boldsymbol{d}}(\alpha)=\left(x_{k l}^{\alpha}\right)$. The group scheme

$$
\mathrm{GL}_{\boldsymbol{d}}=\prod_{i \in Q_{0}} \mathrm{GL}_{d_{i}}
$$

acts on rep ${ }_{Q}^{\boldsymbol{d}}$ by the same formula as above. If $J$ is an ideal in $k Q$, the closed $\mathrm{GL}_{\boldsymbol{d}}{ }^{-}$ subscheme rep $\boldsymbol{d}_{Q, J}^{\boldsymbol{d}}$ is defined by the vanishing of $X_{Q}^{\boldsymbol{d}}(\omega)$ for any $\omega \in \varepsilon_{i} \cdot J \cdot \varepsilon_{j}$, where $i, j$ vary over the set $Q_{0}$.

Now we are ready to define the rank subscheme $\mathcal{C}_{M}$ of $\operatorname{rep}_{Q}^{d}$ associated with a representation $M \in \operatorname{rep}_{Q}^{d}(k)$ : Let $p, q \in \mathbb{N}$, consider two sequences $\left(u_{1}, \ldots, u_{p}\right)$ and $\left(v_{1}, \ldots, v_{q}\right)$ of vertices in $Q_{0}$ and a $p \times q$-matrix $\underline{\omega}=\left(\omega_{i, j}\right)$ such that each $\omega_{i, j}$ belongs to $\varepsilon_{u_{i}} \cdot k Q \cdot \varepsilon_{v_{j}}$. The assignment

$$
N \mapsto N(\underline{\omega})=\left(\begin{array}{lll}
N\left(\omega_{1,1}\right) & \cdots & N\left(\omega_{1, q}\right) \\
\ldots \ldots \ldots \ldots & \ldots \ldots \ldots \\
N\left(\omega_{p, 1}\right) & \cdots & N\left(\omega_{p, q}\right)
\end{array}\right)
$$

leads to a regular morphism

$$
\Theta_{\underline{\omega}}: \operatorname{rep}_{Q}^{d} \rightarrow \mathbb{M}_{p^{\prime} \times q^{\prime}},
$$


where $p^{\prime}=\sum d_{u_{i}}$ and $q^{\prime}=\sum d_{v_{j}}$. We keep track in $\omega_{i, j}$ of the vertices $v_{j}$ and $u_{i}$ even if $\omega_{i, j}=0$. For $M \in \operatorname{rep}_{Q}^{d}(k), \Theta_{\underline{\omega}}(M)$ is a $p^{\prime} \times q^{\prime}$-matrix with coefficients in $k$. We set

$$
\ell_{M, \underline{\omega}}=\Theta_{\underline{\omega}}^{-1}\left(\mathcal{V}_{p^{\prime} \times q^{\prime}}^{\mathrm{rk} \Theta_{\omega}}(M), \quad \ell_{M}=\bigcap \mathcal{C}_{M, \underline{\omega}},\right.
$$

where $\underline{\omega}$ ranges over all possible matrices of paths with all possible sets of starting and terminating vertices. Note that $\boldsymbol{C}_{M, \underline{\omega}}=\operatorname{Spec}\left(k\left[\operatorname{rep}_{Q}^{d}\right] / \mathcal{I}_{M, \underline{\omega}}\right)$, where $\mathcal{I}_{M, \underline{\omega}} \subseteq$ $k\left[\mathrm{rep}_{Q}^{d}\right]$ is the ideal generated by all minors of size $1+\operatorname{rk} \Theta_{\underline{\omega}}(M)$ of the matrix $\Theta_{\underline{\omega}}\left(X_{Q}^{\boldsymbol{d}}\right)$, and that $\mathcal{C}_{M}=\operatorname{Spec}\left(k\left[\operatorname{rep}_{Q}^{\boldsymbol{d}}\right] / \mathcal{I}_{M}\right)$, where $\mathcal{I}_{M}=\sum \mathcal{I}_{M, \underline{\omega}}$. We leave the necessary adjustments for quivers with relations to the reader.

All results presented before in the context of module scheme have a corresponding version in terms of representations of bound quivers $(Q, J)$. The main difference is that instead of finitely generated free presentations of modules we consider projective presentations of representations using the projectives $(k Q / J) \cdot \varepsilon_{i}, i \in Q_{0}$. In particular, if $Q$ is an equioriented Dynkin quiver of type $\mathbb{A}_{n}$,

$$
Q: 1 \stackrel{\alpha_{1}}{\longrightarrow} 2 \stackrel{\alpha_{2}}{\longrightarrow} \cdots \stackrel{\alpha_{n-1}}{\longrightarrow} n,
$$

$J=0, \omega_{j, i}=\alpha_{j-1} \alpha_{j-2} \cdots \alpha_{i}$ and $M$ is a representation in $\operatorname{rep}_{Q}^{d}(k)$, then

$$
\mathcal{I}_{M}=\sum_{1 \leq i<j \leq n} \mathcal{I}_{M,\left(\omega_{j, i}\right)}=\sum_{1 \leq i<j \leq n} I_{1+\mathrm{rk}\left(M\left(\omega_{j, i}\right)\right)}\left(X_{Q}^{\boldsymbol{d}}\left(\omega_{j, i}\right)\right) .
$$

Thus $\mathcal{I}_{M}$ is exactly the ideal generated by determinantal conditions as considered by Lakshmibai and Magyar in [12]. Therefore we can reformulate their main result as follows:

Theorem 4.1. Let $M$ be a representation in $\operatorname{rep}_{Q}^{d}(k)$, where $Q$ is an equioriented Dynkin quiver of type $\mathbb{A}$. Then the ideal $\mathcal{I}_{M}$ is radical and $\mathcal{C}_{M}=\overline{\mathcal{O}}_{M}$.

\section{A geometric version of Morita equivalence for rank schemes}

The purpose of this section is to relate rank schemes for quiver representations to rank schemes for modules over algebras.

Let $A$ be a finite dimensional algebra, and let $S_{1}, \ldots, S_{S}$ be representatives for the isomorphism classes of simple $A$-modules. The Grothendieck group $K_{0}(A)$ can be identified with $\mathbb{Z}^{s}$, and the dimension vector $\operatorname{dim} N \in \mathbb{Z}^{s}$ of a finite dimensional $A$-module $N$ is the vector

$$
\operatorname{dim} N=\left(d_{1}, \ldots, d_{s}\right) \in K_{0}(A),
$$


where $d_{l}$ is the multiplicity of $S_{l}$ in any composition series for $N$. If $e^{l} \in A$ is a primitive idempotent such that $A e^{l}$ is a projective cover for $S_{l}$, we have

$$
d_{l}=\operatorname{dim}_{k} \operatorname{Hom}_{A}\left(A e^{l}, N\right)=\operatorname{rk} N\left(e^{l}\right) .
$$

By [9] or Lemma 1 of [3], there is a connected component $\bmod _{A}^{d}$ of the scheme $\bmod _{A}^{d}$, characterized by the fact that

$$
\bmod _{A}^{d}(k)=\left\{N \in \bmod _{A}^{d}(k): \operatorname{dim} N=\boldsymbol{d}\right\}
$$

for any vector $\boldsymbol{d}=\left(d_{1}, \ldots, d_{s}\right) \in \mathbb{N}^{s}$.

Lemma 5.1. For $M \in \bmod _{A}^{d}(k)$ we have $\ell_{M} \subseteq \bmod _{A}^{d}$.

Proof. As $\bmod _{A}^{\boldsymbol{d}}$ is a connected component in $\bmod _{A}^{d}$, it suffices to show that $\boldsymbol{C}_{M}(k) \subseteq$ $\bmod _{A}^{d}(k)$. Let $N \in \mathcal{C}_{M}(k)$, and set $\operatorname{dim} N=\boldsymbol{d}^{\prime}=\left(d_{1}^{\prime}, \ldots, d_{s}^{\prime}\right)$. Considering the ideal $I_{M,\left(e_{l}\right)}$, where $\left(e^{l}\right)$ is the $1 \times 1$-matrix having the idempotent defined above as its entry, we get that

$$
d_{l}^{\prime}=\operatorname{rk}\left(N\left(e^{l}\right)\right) \leq d_{l}=\mathrm{rk} M\left(e^{l}\right),
$$

for $l=1, \ldots, s$. But

$$
d=\operatorname{dim}_{k} M=\sum_{l=1}^{s} d_{l} \operatorname{dim}_{k} S_{l}=\operatorname{dim}_{k} N=\sum_{l=1}^{s} d_{l}^{\prime} \operatorname{dim}_{k} S_{l},
$$

and thus $\boldsymbol{d}^{\prime}=\boldsymbol{d}$.

Let $B$ be a maximal semisimple subalgebra of $A$. We know that

$$
B \simeq \prod_{l=1}^{s} \mathbb{M}_{n_{l}}(k)
$$

where we set $n_{l}=\operatorname{dim}_{k} S_{l}$. Denote by $e_{i, j}^{l}, l=1, \ldots, s, i, j=1, \ldots, n_{l}$ the canonical basis of $B$, and set $e=\sum_{l=1}^{s} e_{1,1}^{l}$. Then $e A e$ is a basic algebra Morita equivalent to $A$. There is a quiver $Q$ with the set of vertices $\{1, \ldots, s\}$ together with an admissible ideal $J$ in $k Q$ and an algebra isomorphism $\Phi: e A e \rightarrow k Q / J$ such that $\Phi\left(e_{1,1}^{l}\right)=\varepsilon_{l}+J$.

The inclusion $\varphi: B \rightarrow A$ of $k$-algebras induces a regular morphism $\varphi^{d}: \bmod _{A}^{d} \rightarrow$ $\bmod _{B}^{d}$, which restricts to a regular $\mathrm{GL}_{d}$-equivariant $\operatorname{morphism} p: \bmod _{A}^{d} \rightarrow \bmod _{B}^{d}$. Bongartz showed in [3] that the fiber of some special element $E \in \bmod _{B}^{d}$ is isomorphic to rep $\boldsymbol{d}_{Q, J}^{\boldsymbol{d}}$. In fact, he proved that $p$ is a fiber bundle with fiber $p^{-1} E$. We now recall 
his construction and describe explicitly a closed immersion $\eta: \operatorname{rep}_{Q, J}^{d} \rightarrow \bmod _{A}^{d}$ which is an isomorphism onto $p^{-1} E$. First we need some more notation.

Recall that $d=\sum_{l=1}^{s} n_{l} d_{l}$. According to the decomposition

$$
1_{A}=\sum_{l \leq s} \sum_{i \leq n_{l}} e_{i, i}^{l}
$$

into a sum of primitive orthogonal idempotents, we subdivide a $d \times d$-matrix $W$ first into $s^{2}$ "large" blocks, the block $W^{l^{\prime}, l^{\prime \prime}}$ being of size $n_{l^{\prime}} d_{l^{\prime}} \times n_{l^{\prime \prime}} d_{l^{\prime \prime}}, l^{\prime}, l^{\prime \prime} \leq s$, and then we subdivide each block $W^{l^{\prime}, l^{\prime \prime}}$ into $n_{l^{\prime}} n_{l^{\prime \prime}}$ blocks, the block $W_{i, j}^{l^{\prime}, l^{\prime \prime}}$ being of size $d_{l^{\prime}} \times d_{l^{\prime \prime}}, i \leq n_{l^{\prime}}, j \leq n_{l^{\prime \prime}}$. In order to handle these blocks we introduce the obvious injective scheme morphisms

$$
\iota_{i, j}^{l^{\prime}, l^{\prime \prime}}: \mathbb{M}_{d_{l^{\prime}} \times d_{l^{\prime \prime}}} \rightarrow \mathbb{M}_{d}, \quad l^{\prime}, l^{\prime \prime} \leq s, i \leq n_{l^{\prime}}, j \leq n_{l^{\prime \prime}} .
$$

We define a subfunctor $E$ of $\bmod _{B}^{d}$ by $E(R)\left(e_{i, j}^{l}\right)=l_{i, j}^{l, l}\left(1_{n_{l}}\right)$ for a commutative $k$-algebra $R$, where $1_{n}$ denotes the identity matrix in $\mathbb{M}_{n}(R)$. So $E$ is a closed point of the scheme $\bmod _{B}^{d}$. Using the decomposition of an element $a \in A$,

$a=\left(\sum_{l^{\prime} \leq s} \sum_{i \leq n_{l^{\prime}}} e_{i, i}^{l^{\prime}}\right) \cdot a \cdot\left(\sum_{l^{\prime \prime} \leq s} \sum_{j \leq n_{l^{\prime \prime}}} e_{j, j}^{l^{\prime \prime}}\right)=\sum_{l^{\prime}, l^{\prime \prime} \leq s} \sum_{i \leq n_{l^{\prime}}} \sum_{j \leq n_{l^{\prime \prime}}} e_{i, 1}^{l^{\prime}} \cdot\left(e_{1, i}^{l^{\prime}} \cdot a \cdot e_{j, 1}^{l^{\prime \prime}}\right) \cdot e_{1, j}^{l^{\prime \prime}}$,

and the fact that $e_{1, i}^{l^{\prime}} \cdot a \cdot e_{j, 1}^{l^{\prime \prime}}$ belongs to $e A e$, we define the scheme morphism

$$
\eta: \operatorname{rep}_{Q, J}^{d} \rightarrow \bmod _{A}^{d}, \quad(\eta N)(a)=\sum_{l^{\prime}, l^{\prime \prime} \leq s} \sum_{i \leq n_{l^{\prime}}} \sum_{j \leq n_{l^{\prime \prime}}} l_{i, j}^{l^{\prime}, l^{\prime \prime}}\left(N\left(\Phi\left(e_{1, i}^{l^{\prime}} \cdot a \cdot e_{j, 1}^{l^{l^{\prime \prime}}}\right)\right)\right) .
$$

Then $\eta$ is an isomorphism onto $p^{-1}(E)$. Note that if we view elements of $\operatorname{rep}_{Q, J}^{d}(k)$ and $\bmod _{A}^{d}(k)$ as representations and modules, respectively, then the map

$$
\eta(k): \operatorname{rep}_{Q, J}^{\boldsymbol{d}}(k) \rightarrow \bmod _{A}^{\boldsymbol{d}}(k)
$$

is in accordance with an equivalence between the category of representations of $(Q, J)$ and the category of $A$-modules.

Proposition 5.2. With the above notations we have

$$
\eta^{-1}\left(e_{\eta M}\right)=e_{M} \subseteq \operatorname{rep}_{Q, J}^{d}
$$

for any $M \in \operatorname{rep}_{Q, J}^{d}(k)$.

Proof. The result is a consequence of the following two facts. 
(1) For any $p \times q$-matrix $\underline{a}$ with coefficients in $A$ there are $p^{\prime}+q^{\prime}$ vertices $u_{1}, \ldots, u_{p^{\prime}}$, $v_{1}, \ldots, v_{q^{\prime}}$ of $Q$ and elements $\omega_{i^{\prime}, j^{\prime}} \in \varepsilon_{u_{i^{\prime}}} \cdot k Q / J \cdot \varepsilon_{v_{j^{\prime}}}$ yielding $\underline{\omega}(\underline{a})=\left(\omega_{i^{\prime}, j^{\prime}}\right)$ such that

$$
e_{M, \underline{\omega}(a)}=\eta^{-1} \boldsymbol{C}_{\eta M, \underline{a}} .
$$

(2) For any $p^{\prime}, q^{\prime}$, any vertices $u_{1}, \ldots, u_{p^{\prime}}, v_{1}, \ldots, v_{q^{\prime}}$ of $Q$ and any elements $\omega_{i^{\prime}, j^{\prime}} \in \varepsilon_{u_{i^{\prime}}} \cdot k Q / J \cdot \varepsilon_{v_{j^{\prime}}}$ with $\underline{\omega}=\left(\omega_{i^{\prime}, j^{\prime}}\right)$, there is a $p^{\prime} \times q^{\prime}$-matrix $\underline{a}(\underline{\omega})$ with coefficients in $A$ such that

$$
\varphi_{M, \underline{\omega}}=\eta^{-1} \varphi_{\eta M, \underline{a}(\omega)} .
$$

In order to prove (1) we first construct $\underline{\omega}(a)$ for $a \in A$ such that $N(\underline{\omega}(a))=$ $(\eta N)(a)$ for $N \in \operatorname{rep}_{Q, J}^{d}$ : We set $p^{\prime}=q^{\prime}=n=\sum_{l=1}^{s} n_{l}$, choose $u_{(l, i)}=v_{(l, i)}=$ $l \in Q_{0}$ for $l=1, \ldots, s, i=1, \ldots, n_{s}$, and set $\underline{\omega}=\left(\omega_{\left(l^{\prime}, i\right),\left(l^{\prime \prime}, j\right)}\right)$ with

$$
\omega_{\left(l^{\prime}, i\right),\left(l^{\prime \prime}, j\right)}(a)=\Phi\left(e_{1, i}^{l^{\prime}} \cdot a \cdot e_{j, 1}^{l^{\prime \prime}}\right) \in \varepsilon_{l^{\prime}} \cdot k Q / J \cdot \varepsilon_{l^{\prime \prime}} .
$$

By the definition of $\eta$, we have

$$
(\eta N)(a)=\sum_{l^{\prime}, l^{\prime \prime} \leq s} \sum_{i \leq n_{l^{\prime}}} \sum_{j \leq n_{l^{\prime \prime}}} l_{i, j}^{l^{\prime}, l^{\prime \prime}}\left(N\left(\Phi\left(e_{1, i}^{l^{\prime}} \cdot a \cdot e_{j, 1}^{l^{\prime \prime}}\right)\right)\right)=N(\underline{\omega}(a)) .
$$

as desired. For a $p \times q$-matrix $\underline{a}=\left(a_{i^{\prime}, j^{\prime}}\right)$ with coefficients in $A$, we set $\underline{\omega}(\underline{a})=$ $\left(\underline{\omega}\left(a_{i^{\prime}, j^{\prime}}\right)\right)$. As above, we conclude that

$$
(\eta N)(\underline{a})=N(\underline{\omega}(\underline{a})) .
$$

In particular, $r=1+\operatorname{rk}(\eta M)(\underline{a})=1+\operatorname{rk} M(\underline{\omega}(\underline{a}))$. As a consequence we obtain that, for any commutative $k$-algebra $R, I_{r}(N(\underline{\omega}(\underline{a})))=0$ if and only if $I_{r}((\eta N)(\underline{a}))=0$, for any $N \in \operatorname{rep}_{Q, J}^{d}(R)$, which is equivalent to $\mathcal{C}_{M, \underline{\omega}(\underline{a})}(R)=$ $\left(\eta^{-1} C_{\eta M, \underline{a}}\right)(R)$.

For the proof of (2), we set $\underline{a}(\underline{\omega})=\left(\Phi^{-1} \omega_{i^{\prime}, j^{\prime}}\right)$ for $\underline{\omega}=\left(\omega_{i^{\prime}, j^{\prime}}\right)$. For any commutative $k$-algebra $R$ and any $N \in \operatorname{rep}_{Q, J}^{d}(R)$, the only possibly non-zero entries of the $d \times d$-matrix $(\eta N)\left(\Phi^{-1} \omega_{i^{\prime}, j^{\prime}}\right)=i_{1,1}^{u_{i^{\prime}}, v_{j^{\prime}}}\left(N\left(\omega_{u_{i^{\prime}}, v_{j^{\prime}}}\right)\right)$ sit in the small block in the upper left corner of the big block corresponding to $l^{\prime}=u_{i^{\prime}}, l^{\prime \prime}=v_{j^{\prime}}$. Therefore the rows and the columns of the $p^{\prime} d \times q^{\prime} d$-matrix $(\eta N)(\underline{a}(\underline{\omega}))$ can be permuted in such a way that the upper left corner becomes $N(\underline{\omega})$ and all other entries are zero. In other words, there are invertible matrices, in fact permutation matrices, $U$ and $V$ such that

$$
U \cdot(\eta N)(\underline{a}(\underline{\omega})) \cdot V=\left(\begin{array}{cc}
N(\underline{\omega}) & 0 \\
0 & 0
\end{array}\right) .
$$

Then clearly $r=1+\operatorname{rk}(\eta M)(\underline{a}(\underline{\omega}))=1+\operatorname{rk} M(\underline{\omega})$ and by Lemma 2.2 we have

$$
I_{r}((\eta N)(\underline{a}(\underline{\omega})))=I_{r}\left(\begin{array}{cc}
N(\underline{\omega}) & 0 \\
0 & 0
\end{array}\right)=I_{r}(N(\underline{\omega})) .
$$


Therefore $I_{r}(N(\underline{\omega}))=0$ if and only if $I_{r}((\eta N)(\underline{a}(\underline{\omega})))=0$, and we conclude that $\ell_{M, \underline{\omega}}(R)=\left(\eta^{-1} \boldsymbol{C}_{\eta M, \underline{a}(\omega)}\right)(R)$.

Following Hesselink (see (1.7) in [11]) we call two pointed schemes $\left(\mathcal{X}, x_{0}\right)$ and $\left(\mathcal{Y}, y_{0}\right)$ smoothly equivalent if there are smooth morphisms $f: \mathcal{Z} \rightarrow \mathcal{X}, g: \mathcal{Z} \rightarrow \mathcal{Y}$ sending a point $z_{0} \in \mathcal{Z}$ to $x_{0}$ and $y_{0}$, respectively. This is an equivalence relation and an equivalence class will be denoted by $\operatorname{Sing}\left(\mathcal{X}, x_{0}\right)$ and called the type of singularity of $\mathcal{X}$ at $x_{0}$. Assuming $\operatorname{Sing}\left(\mathcal{X}, x_{0}\right)=\operatorname{Sing}\left(\mathcal{Y}, y_{0}\right)$, the scheme $\mathcal{X}$ is regular (or reduced, normal, Cohen-Macaulay, respectively) at $x_{0}$ if and only if the same is true for the scheme $y$ at $y_{0}$ (see [10], Section 17, for more information about smooth morphisms).

Theorem 5.3. Let $\eta: \operatorname{rep}_{Q, J}^{d} \rightarrow \bmod _{A}^{d}$ be the morphism defined above. Suppose $M$ and $M^{\prime}$ in $\operatorname{rep}_{Q, J}^{d}(k)$ are such that $M^{\prime}$ belongs to $\mathcal{C}_{M}(k)$. Then $\eta M^{\prime}$ belongs to $\mathcal{C}_{\eta M}(k)$ and

$$
\operatorname{Sing}\left(\mathcal{C}_{\eta M}, \eta M^{\prime}\right)=\operatorname{Sing}\left(\mathcal{C}_{M}, M^{\prime}\right)
$$

Proof. The orbit map $\psi: \mathrm{GL}_{d} \rightarrow \bmod _{B}^{\boldsymbol{d}}$ defined by $\psi(g)=g * E$ is smooth and induces an isomorphism of schemes $\mathrm{GL}_{d} / \mathrm{GL}_{\boldsymbol{d}} \rightarrow \bmod _{B}^{d}$, where $\mathrm{GL}_{\boldsymbol{d}}=\prod_{l=1}^{s} \mathrm{GL}_{d_{l}}$ is embedded into $\mathrm{GL}_{d}$ via

$$
\left(g_{1}, \ldots, g_{s}\right) \mapsto \sum_{l=1}^{s} \sum_{i=1}^{n_{l}} l_{i, i}^{l, l}\left(g_{l}\right) .
$$

It is not hard to see (compare e.g. [6]) that the diagram

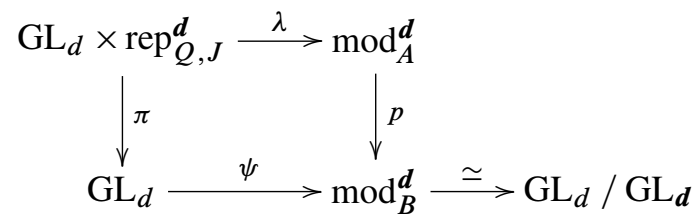

is a pullback, where $\pi$ is the projection to the first factor and $\lambda(g, N)=g * \eta N$. Note that $\lambda$ is smooth as smoothness is preserved under base change. As $\lambda(k)$ is surjective and thus contains $\eta M^{\prime}$ in its image, it is enough to show that $\lambda^{-1} \mathscr{C}_{\eta M}=\mathrm{GL}_{d} \times \mathscr{C}_{M}$.

A pair $(g, N) \in \mathrm{GL}_{d}(R) \times \mathcal{C}_{\eta M}(R)$ belongs to $\left(\lambda^{-1} \boldsymbol{C}_{\eta M}\right)(R)$, for a commutative $k$-algebra $R$, if and only if $g * \eta N \in \mathcal{C}_{\eta M}(R)$. As by Lemma $2.2 \mathcal{C}_{\eta M}(R)$ is stable under $\mathrm{GL}_{d}(R)$, this is equivalent to $\eta N \in \mathcal{C}_{\eta M}(R)$, which is in turn equivalent to $(g, N) \in \mathrm{GL}_{d}(R) \times \mathcal{C}_{M}(R)$ by Proposition 5.2. 


\section{Hom-controlled exact functors}

Let $\varphi: A \rightarrow B$ be a homomorphism of finite dimensional algebras and $\varphi^{*}: \bmod B \rightarrow$ $\bmod A$ the induced change of scalars functor. For a $B$-module $M$ we will use the notation $M={ }_{B} M$ and $\varphi^{*}(M)={ }_{A} M$. Thus $\varphi^{d}\left(\mathcal{O}_{B} M\right)=\mathcal{O}_{A} M$ for any module $M$ in $\bmod _{B}^{d}(k)$.

Following [17], we call an exact functor $\mathscr{F}: \bmod B \rightarrow \bmod A$ hom-controlled, if there is a bilinear form $\xi: K_{0}(B) \times K_{0}(B) \rightarrow \mathbb{Z}$ such that

$$
[\mathcal{F} U, \mathcal{F} V]_{A}-[U, V]_{B}=\xi(\operatorname{dim} U, \operatorname{dim} V)
$$

for any $U, V \in \bmod B$. Here and later on, we abbreviate $\operatorname{dim}_{k} \operatorname{Hom}_{B}(U, V)$ by $[U, V]_{B}$, for any $U, V \in \bmod B$ and similarly for $A$-modules.

Assume now that the functor $\varphi^{*}$ is hom-controlled. It follows from Theorem 1.1 of [17] that the restriction of $\varphi^{d}$

$$
\overline{\mathcal{O}}_{B} M \rightarrow \overline{\mathcal{O}}_{A} M
$$

is a smooth morphism. The aim of this section is to show this is still true if we replace the orbit closures by the rank schemes $\ell_{B} M$ and $\ell_{A} M$.

Let $L$ be a finite dimensional $A$-module and $t \in \mathbb{N}$. We choose a $p \times q$-matrix $\underline{a}$ such that $\operatorname{Coker}\left(v_{\underline{a}}\right)$ is $A$-isomorphic to $L$. Let $\bmod _{A, L, t}^{d}$ be the closed subscheme of $\bmod _{A}^{d}$ defined by the ideal $I_{1+q d-t}\left(X_{A}^{d}(\underline{a})\right)$ in $k\left[\bmod _{A}^{d}\right]$. The proof of Lemma 3.2 can easily be generalized to show that this ideal is determined uniquely by $t$ and the isomorphism class of $L$. By $\left(\bmod _{A, L, t}^{d}\right)^{0}$ we denote the open subscheme of $\bmod _{A, L, t}^{d}$ whose $k$-points are the modules $N$ with $[L, N]_{A}=t$.

It has been proved in Section 4 of [17] that $\varphi^{d}$ restricts to a smooth morphism from $\bmod _{B}^{d}$ to $\left(\bmod _{A, A_{A} B, t}^{d}\right)^{0}$ for any $\boldsymbol{d} \in K_{0}(B)$, where $d$ is the common dimension of all modules in $\bmod _{B}^{d}$ and $t=d+\xi(\operatorname{dim} B, \boldsymbol{d})$. We denote by

$$
\psi: \bmod _{B}^{d} \rightarrow \bmod _{A, A}^{d} B, t
$$

the composition of this morphism with the open immersion into $\bmod _{A, A}^{d} B, t$, which is still smooth.

Theorem 6.1. Let $\varphi: A \rightarrow B$ be an algebra homomorphism such that $\varphi^{*}$ is a homcontrolled exact functor and fix $M \in \bmod _{B}^{d}(k)$. Then the morphism $\psi$ restricts to a morphism

$$
\ell_{B} M \rightarrow \ell_{A} M,
$$

which is smooth.

Proof. We know that $\ell_{B} M$ is a subscheme of $\bmod _{B}^{d}$. Thus the claim will be proved if we can show that $\ell_{A} M$ is a subscheme of $\bmod _{A, A}^{d} B, t$, where $t=d+\xi(\operatorname{dim} B, \boldsymbol{d})$, 
and that $\psi^{-1}\left(\ell_{A} M\right)=\ell_{B} M$, or equivalently that

$$
\left(\varphi^{d}\right)^{-1} \bigodot_{A} \cap \bmod _{B}^{d}=\bigodot_{B} M
$$

The first part is easy, because

$$
\left[{ }_{A} B,{ }_{A} M\right]_{A}=\left[{ }_{B} B,{ }_{B} M\right]_{B}+\xi(\operatorname{dim} B, \operatorname{dim} M)=t
$$

and consequently, $I_{1+q d-t}\left(X_{A}^{d}(\underline{a})\right)=\mathcal{I}_{A} M_{A} B$, where $\underline{a}$ is a $p \times q$-matrix with coefficients in $A$ and with ${ }_{A} B=\operatorname{Coker} v_{\underline{a}}$. The inclusion $\bigodot_{B} M \subseteq\left(\varphi^{d}\right)^{-1} \bigodot_{A} M \cap$ $\bmod _{B}^{d}$ follows from Lemma 3.5 and Lemma 5.1. In order to prove the reverse inclusion we will show that, for any $B$-module ${ }_{B} L$, we have

$$
\mathcal{I}_{B} M,_{B} L \subseteq k\left[\bmod _{B}^{d}\right] \cdot\left(\varphi^{d}\right)^{*} \mathcal{I}_{A} M,_{A} L+\mathcal{I}\left(\bmod _{B}^{d}\right)
$$

in $k\left[\bmod _{B}^{d}\right]$, where $\mathcal{I}\left(\bmod _{B}^{\boldsymbol{d}}\right)$ is the ideal defining $\bmod _{B}^{\boldsymbol{d}}$.

Let $L$ be a finite dimensional $B$-module. Choosing a finite free presentation of ${ }_{A} L$ we obtain the exact sequence of $A$-modules

$$
A^{p} \stackrel{v_{a}}{\rightarrow} A^{q} \rightarrow{ }_{A} L \rightarrow 0
$$

for some $p, q \geq 1$ and a $p \times q$-matrix $\underline{a}$ with coefficients in $A$. We apply the tensor functor $B \otimes_{A}(-)$ to get another exact sequence

$$
B^{p} \stackrel{\nu_{\varphi(\underline{a})}}{\longrightarrow} B^{q} \rightarrow B \otimes_{A} L \rightarrow 0
$$

Using the homomorphism $\varphi$ we have a left and a right $A$-module structure on $B$, and the functor $\varphi^{*}$ can be identified with the functor ${ }_{A} B \otimes_{B}(-)$ as well as with $\operatorname{Hom}_{B}\left({ }_{B} B_{A},-\right)$. Observe that

$$
B \otimes_{A} L=B \otimes_{A} \varphi^{*}\left({ }_{B} L\right) \simeq B \otimes_{A} B \otimes_{B} L=\Omega \otimes_{B} L,
$$

where $\Omega$ is the $B$-B-bimodule $B \otimes_{A} B$, and that for any $B$-module $Y$ we have

$$
\begin{aligned}
\operatorname{Hom}_{A}\left({ }_{A} L,{ }_{A} Y\right) & =\operatorname{Hom}_{A}\left({ }_{A} B \otimes_{B} L, \operatorname{Hom}_{B}\left({ }_{B} B_{A},{ }_{B} Y\right)\right) \\
& \simeq \operatorname{Hom}_{B}\left({ }_{B} B \otimes_{A} B \otimes_{B} L,{ }_{B} Y\right) \\
& =\operatorname{Hom}_{B}\left(\Omega \otimes_{B} L,{ }_{B} Y\right) .
\end{aligned}
$$

As $\varphi^{*}$ is hom-controlled, we obtain

$$
\left[\Omega \otimes_{B} L, Y\right]_{B}-[L, Y]_{B}=\xi(\operatorname{dim} L, \operatorname{dim} Y)
$$

for any $B$-module $Y$.

Let $\left\{P_{1}, \ldots, P_{n}\right\}$ be a complete set of pairwise non-isomorphic indecomposable projective $B$-modules and $S_{i}=P_{i} / \operatorname{rad}\left(P_{i}\right)$ for $i \leq n$. Note that $\left\{S_{1}, \ldots, S_{n}\right\}$ is a complete set of pairwise non-isomorphic simple $B$-modules. 
Let $s_{i}=\xi\left(\operatorname{dim} L, \operatorname{dim} S_{i}\right)$ for $i \leq n$ and $P_{L}=\bigoplus_{i} P_{i}^{s_{i}}$, and let $y_{i}$ denote the $i$-th coordinate of $\operatorname{dim} Y$. Then

$$
\begin{aligned}
{\left[P_{L}, Y\right] } & =\sum_{i=1}^{n} s_{i} \cdot\left[P_{i}, Y\right]=\sum_{i=1}^{n} \xi\left(\operatorname{dim} L, \operatorname{dim} S_{i}\right) \cdot y_{i} \\
& =\xi\left(\operatorname{dim} L, \operatorname{dim}\left(\bigoplus_{i=1}^{n} S_{i}^{y_{i}}\right)\right)=\xi(\operatorname{dim} L, \operatorname{dim} Y),
\end{aligned}
$$

and consequently,

$$
\left[\Omega \otimes_{B} L, Y\right]=\left[L \oplus P_{L}, Y\right] .
$$

The latter holds for any finite dimensional $B$-module $Y$, hence $\Omega \otimes_{B} L \simeq L \oplus P_{L}$, by Auslander's theorem. This implies that the ideal generated by $\left(\varphi^{d}\right)^{*} \mathcal{I}_{A} M_{,_{A} L}$ equals $\mathcal{I}_{B} M,_{B}\left(L \oplus P_{L}\right)$.

If $P_{L}=0$, the inclusion (6.1) clearly holds. Otherwise, choose matrices $\underline{b}^{\prime}$ and $\underline{b}^{\prime \prime}$ with coefficients in $B$ such that the $\operatorname{Coker}\left(v_{b^{\prime}}\right) \simeq L$ and $\operatorname{Coker}\left(v_{b^{\prime \prime}}\right) \simeq P_{L}$. Let $r_{1}=\operatorname{rk} M\left(\underline{b}^{\prime}\right), r_{2}=\operatorname{rk} M\left(\underline{b}^{\prime \prime}\right)$, and set $X=X_{B}^{\underline{d}}$. Then

$$
\tilde{I}_{B} M,_{B}\left(L \oplus P_{L}\right)=I_{1+r_{1}+r_{2}}\left(\begin{array}{cc}
X\left(\underline{b}^{\prime}\right) & 0 \\
0 & X\left(\underline{b}^{\prime \prime}\right)
\end{array}\right) \supseteq I_{1+r_{1}}\left(X\left(\underline{b}^{\prime}\right)\right) \cdot I_{r_{2}}\left(X\left(\underline{b}^{\prime \prime}\right)\right),
$$

by Lemma 2.3. Obviously $I_{1+r_{1}}\left(X\left(\underline{b}^{\prime}\right)\right)=\mathcal{I}_{B} M,_{B} L$ and therefore

$$
\mathcal{I}_{B} M,_{B}\left(L \oplus P_{L}\right)+\mathcal{I}\left(\bmod _{B}^{\boldsymbol{d}}\right) \supseteq \mathcal{I}_{B} M,_{B} L \cdot\left(I_{r_{2}}\left(X\left(\underline{b}^{\prime \prime}\right)\right)+\mathcal{I}\left(\bmod _{B}^{\boldsymbol{d}}\right)\right) .
$$

Thus it suffices to show that

$$
\left.I_{r_{2}}\left(X \underline{b}^{\prime \prime}\right)\right)+\mathcal{I}\left(\bmod _{B}^{d}\right)=k\left[\bmod _{B}^{d}\right] .
$$

Let $N$ be a module in $\bmod _{B}^{d}(k)$. The condition that $I_{r_{2}}\left(X\left(\underline{b}^{\prime \prime}\right)\right)$ vanishes on $N$ means $\left[P_{L}, N\right]>\left[P_{L}, M\right]$ while $\mathcal{I}\left(\bmod _{B}^{d}\right)$ vanishes on $N$ if and only if $\operatorname{dim} N=$ $\operatorname{dim} M$. Since $\operatorname{dim} N=\operatorname{dim} M$ implies $\left[P_{L}, N\right]=\left[P_{L}, M\right]$, there exists no point $N$ on which the ideal $I_{r_{2}}\left(X\left(\underline{b}^{\prime \prime}\right)\right)+\mathcal{I}\left(\bmod _{B}^{d}\right)$ vanishes.

Theorem 1.2 in [17] says that

$$
\operatorname{Sing}\left(\overline{\mathcal{O}}_{\mathcal{F} M}, \mathscr{F} M^{\prime}\right)=\operatorname{Sing}\left(\overline{\mathcal{O}}_{M}, M^{\prime}\right)
$$

for a hom-controlled exact functor $\mathcal{F}, M \in \bmod _{A}^{d}(k)$ and $M^{\prime} \in \overline{\mathcal{O}}_{M}$. The proof can be adapted to rank schemes, yielding the following result. The only slight difficulty is taken care of by the lemma following the theorem. Recall that if $M^{\prime}$ is a point of $\mathcal{C}_{M}(k)$, the modules $M$ and $M^{\prime}$ have the same dimension vector, and so do their images $\mathcal{F}(M)$ and $\mathcal{F}\left(M^{\prime}\right)$ under an exact functor. 
Theorem 6.2. Let $\mathcal{F}: \bmod B \rightarrow \bmod A$ be a hom-controlled exact functor and fix $M, M^{\prime} \in \bmod _{B}^{d}(k)$ with $M^{\prime} \in \overline{\mathcal{O}}_{M}$. Let $\boldsymbol{e}$ be the common dimension vector of $\mathcal{F} M$ and $\mathscr{F} M^{\prime}$. Identifying $\mathscr{F} M$ and $\mathscr{F} M^{\prime}$ with the corresponding elements in $\bmod _{A}^{e}(k)$ we obtain that $\mathscr{F} M^{\prime} \in \mathcal{C}_{\mathscr{F} M}$ and

$$
\operatorname{Sing}\left(\mathcal{C}_{\mathscr{F} M}, \mathscr{F} M^{\prime}\right)=\operatorname{Sing}\left(\mathcal{C}_{M}, M^{\prime}\right) .
$$

Lemma 6.3. Let $B=C \times D$ be the product of an algebra $C$ with a semisimple algebra $D$, both finite dimensional, fix a $B$-module $M=\left(M_{1}, M_{2}\right)$, and choose $M^{\prime}=\left(M_{1}^{\prime}, M_{2}^{\prime}\right) \in \mathcal{C}_{M}(k)$. Then we have

$$
\operatorname{Sing}\left(\mathcal{C}_{M}, M^{\prime}\right)=\operatorname{Sing}\left(\mathcal{C}_{M_{1}}, M_{1}^{\prime}\right) .
$$

Proof. The easiest way to see this is to replace the algebras by quivers and relations using Theorem 5.3. Then we have

$$
\ell_{M}=\ell_{M_{1}} \times \ell_{M_{2}}, \ell_{M^{\prime}}=\ell_{M_{1}^{\prime}} \times \ell_{M_{2}^{\prime}}
$$

As $D$ is semisimple, its quiver consists of some vertices but no arrows, and thus $\ell_{M_{2}}=\ell_{M_{2}^{\prime}}=\left\{M_{2}\right\}=\left\{M_{2}^{\prime}\right\}$.

The above theorem remains true if modules are replaced by representations of quivers, by Theorem 5.3. In particular, applying the theorem to the exact functors constructed in [1] and [2] we may generalize Theorem 4.1 as follows.

Theorem 6.4. Let $M$ be a representation in $\operatorname{rep}_{Q}^{d}(k)$, where $Q$ is a Dynkin quiver of type $\mathbb{A}$. Then the ideal $\mathcal{I}_{M}$ is radical and $\mathcal{C}_{M}=\overline{\mathcal{O}}_{M}$.

Let us describe the ideal $\mathcal{I}_{M}$ explicitly. We know from Corollary 3.6 that $\mathcal{I}_{M}=$ $\sum \mathcal{I}_{M, L}$, where $L$ ranges over all indecomposable representations of $Q$. Suppose the underlying graph of $Q$ is

$$
1-2-\cdots-n .
$$

Independently of the orientations of the arrows, an indecomposable $L$ is given by an interval $L=\left[l, l^{\prime}\right]$ in $[1, n]$, for some $l \leq l^{\prime}$ : Each vertex in $\left[l, l^{\prime}\right]$ is represented by $k$, each arrow between such vertices by the matrix (1). Denote the full subquiver of $Q$ with vertex set $\left[l, l^{\prime}\right]$ by $Q_{\left[l, l^{\prime}\right]}$. We associate with $L$ the sequence $l \leq v_{1}<\cdots<$ $v_{q} \leq l^{\prime}$ of all sources of $Q_{\left[l, l^{\prime}\right]}$ and the sequence $l-1 \leq u_{1}<\cdots<u_{p} \leq l^{\prime}+1$ consisting of all sinks in $Q_{\left[l, l^{\prime}\right]}$ distinct from $l, l^{\prime}$ in addition to

$$
\begin{cases}l-1 & \text { if } 1<l \text { and there is an arrow } l-1 \leftarrow l \in Q_{1}, \\ l^{\prime}+1 & \text { if } l^{\prime}<n \text { and there is an arrow } l^{\prime} \rightarrow l^{\prime}+1 \in Q_{1} .\end{cases}
$$


For any $u_{i}$ there is either some $v_{j^{\prime}}<u_{i}$ and a path $\omega_{i, j^{\prime}}: v_{j^{\prime}} \rightarrow u_{i}$ in $Q$ or some $v_{j^{\prime \prime}}>u_{i}$ and a path $\omega_{i, j^{\prime \prime}}: v_{j^{\prime \prime}} \rightarrow u_{i}$ in $Q$ or both, in which case we must have $j^{\prime \prime}=j^{\prime}+1$. The $p \times q$-matrix $\underline{\omega}$ corresponding to $L=\left[l, l^{\prime}\right]$ has all its entries 0 , except for those just described.

In the special case

$$
Q=1 \stackrel{\alpha_{1}}{\longrightarrow} 2 \ll \alpha_{2}{ }^{\alpha_{2}} \stackrel{\alpha_{3}}{\longleftarrow} \stackrel{\alpha_{4}}{\longrightarrow} 5,
$$

the matrices to be considered are

$$
\begin{array}{ccc}
\left(\alpha_{1}\right), & \left(\alpha_{2}\right), \quad\left(\alpha_{3}\right), & \left(\alpha_{4}\right), \\
\left(\begin{array}{ll}
\alpha_{1} & \alpha_{2}
\end{array}\right), & \left(\begin{array}{ll}
\alpha_{2} \circ \alpha_{3}
\end{array}\right), & \left(\begin{array}{l}
\alpha_{3} \\
\alpha_{4}
\end{array}\right), \\
\left(\begin{array}{ll}
\alpha_{1} & \alpha_{2} \circ \alpha_{3}
\end{array}\right), & \left(\begin{array}{cc}
\alpha_{2} \circ \alpha_{3} \\
\alpha_{4}
\end{array}\right), & \left(\begin{array}{cc}
\alpha_{1} & \alpha_{2} \circ \alpha_{3} \\
0 & \alpha_{4}
\end{array}\right) .
\end{array}
$$

\section{Tangent spaces}

Let $N$ belong to $\ell_{M}(k)$. The main aim of this section is to describe the tangent space $\mathcal{T}_{e_{M}, N}$ in terms of selfextensions of the module $N$.

The tangent space $\mathcal{T}_{\bmod _{A}^{d}, N}$ can be identified with the space of 1-cocycles $\mathbb{Z}_{A}^{1}(N, N)$, that is, with the set of $k$-linear maps $Z: A \rightarrow \mathbb{M}_{d}(k)$ with the property that $Z\left(a_{1} a_{2}\right)=N\left(a_{1}\right) Z\left(a_{2}\right)+Z\left(a_{1}\right) N\left(a_{2}\right)$ for any $a_{1}, a_{2} \in A$. Note that from a 1 -cocycle $Z$ we obtain a module structure on $k^{d} \oplus k^{d}$ given by

$$
\left(\begin{array}{cc}
N & Z \\
0 & N
\end{array}\right)(a)=\left(\begin{array}{cc}
N(a) & Z(a) \\
0 & N(a)
\end{array}\right)
$$

and that the sequence

$$
\varphi(Z): 0 \rightarrow N \stackrel{\left(\begin{array}{l}
1 \\
0
\end{array}\right)}{\longrightarrow}\left(\begin{array}{cc}
N & Z \\
0 & N
\end{array}\right) \stackrel{\left(\begin{array}{ll}
0 & 1
\end{array}\right)}{\longrightarrow} N \rightarrow 0
$$

is exact.

The tangent space $\mathcal{T}_{\mathcal{O}_{N}, N}$ can be identified with the space of 1-coboundaries $\mathbb{B}_{A}^{1}(N, N)=\left\{h \cdot N-N \cdot h ; h \in \mathbb{M}_{d}(k)\right\}$. By [9], Proposition 1.1, the map $\varphi$ induces an isomorphism, called Voigt's isomorphism,

$$
\mathcal{T}_{\bmod _{A}^{d}, N} / \mathcal{T}_{\mathcal{O}_{N}, N} \simeq \mathbb{Z}_{A}^{1}(N, N) / \mathbb{B}_{A}^{1}(N, N)=\operatorname{Ext}_{A}^{1}(N, N) .
$$

Since $\mathcal{T}_{\mathcal{O}_{N}, N} \subseteq \mathcal{T}_{\mathscr{C}_{M}, N} \subseteq \mathcal{T}_{\bmod _{A}^{d}, N}$, the tangent space $\mathcal{T}_{\mathscr{C}_{M}, N}$ corresponds to a subspace of $\mathbb{Z}_{A}^{1}(N, N)$ containing $\mathbb{B}_{A}^{1}(N, N)$, which we now describe. 
Let $\mathcal{F}$ and $\mathcal{F}^{\prime}$ be complete sets of pairwise non-isomorphic indecomposable modules $X$ and $X^{\prime}$ such that $[N, X]=[M, X]$ and $\left[X^{\prime}, N\right]=\left[X^{\prime}, M\right]$, respectively. Set

$\mathcal{E}(Y, Z)$

$$
\begin{aligned}
& =\left\{[\sigma: 0 \rightarrow Z \rightarrow W \rightarrow Y \rightarrow 0]_{\sim} \in \operatorname{Ext}_{A}^{1}(Y, Z) ; \delta_{\sigma}(X)=0 \text { for all } X \in \mathcal{F}\right\} \\
& =\left\{[\sigma: 0 \rightarrow Z \rightarrow W \rightarrow Y \rightarrow 0]_{\sim} \in \operatorname{Ext}_{A}^{1}(Y, Z) ; \delta_{\sigma}^{\prime}\left(X^{\prime}\right)=0 \text { for all } X^{\prime} \in \mathcal{F}^{\prime}\right\} .
\end{aligned}
$$

for two $A$-modules $Y, Z$, where

$$
\begin{aligned}
\delta_{\sigma}(X) & =\operatorname{dim}_{k} \operatorname{Hom}_{A}(Z \oplus Y, X)-\operatorname{dim}_{k} \operatorname{Hom}_{A}(W, X), \\
\delta_{\sigma}^{\prime}\left(X^{\prime}\right) & =\operatorname{dim}_{k} \operatorname{Hom}_{A}\left(X^{\prime}, Y \oplus Z\right)-\operatorname{dim}_{k} \operatorname{Hom}_{A}\left(X^{\prime}, W\right) .
\end{aligned}
$$

Note that the pushout or pullback of an exact sequence in $\mathscr{E}$ belongs to $\mathcal{E}$ again. As a consequence, $\mathcal{E}(-,-)$ is a $k$-subfunctor of $\operatorname{Ext}_{A}^{1}(-,-)$.

Proposition 7.1. For $N \in \mathcal{C}_{M}(k)$, Voigt's isomorphism restricts to an isomorphism

$$
\mathcal{T}_{e_{M}, N} / \mathcal{T}_{\mathcal{O}_{N}, N} \simeq \mathcal{E}(N, N) .
$$

The following corollary is an immediate consequence.

Corollary 7.2. Let $N$ be a point of $\overline{\mathcal{O}}_{M}$. Then $\operatorname{codim}(M, N) \leq \operatorname{dim}_{k} \mathcal{E}(N, N)$, and equality holds if and only if $N$ is a regular point of $\mathcal{C}_{M}$.

By definition, $\operatorname{codim}(M, N)=\operatorname{dim} \mathcal{O}_{M}-\operatorname{dim} \mathcal{O}_{N}$.

We will prove Proposition 7.1 in several steps. We begin by characterizing the tangent space to the scheme $\mathcal{V}_{p \times q}^{r}$ at some matrix $N \in \mathcal{V}_{p \times q}^{r}(k)$ as a subspace of the tangent space of $\mathbb{M}_{p \times q}$ at $N$, which we identify with $\mathbb{M}_{p \times q}(k)$.

Lemma 7.3. Fix $r \leq p, q$, and choose a matrix $N \in \mathcal{V}_{p \times q}^{r}(k)$. Then

$$
\mathcal{T}_{\mathcal{V}_{p \times q}^{r}, N}= \begin{cases}\mathbb{M}_{p \times q}(k) & \text { if } \operatorname{rk} N<r, \\
\left\{D \in \mathbb{M}_{p \times q}(k) ; \operatorname{rk}\left(\begin{array}{cc}
N & D \\
0 & N
\end{array}\right)=2 r\right\} & \text { if } \operatorname{rk} N=r .\end{cases}
$$

Proof. The algebraic group scheme $\mathrm{GL}_{p} \times \mathrm{GL}_{q}$ acts on $\mathbb{M}_{p \times q}$ via $(g, h) * N^{\prime}=$ $g \cdot N^{\prime} \cdot h^{-1}$, and we know that $N=g \cdot\left(\begin{array}{cc}1_{s} & 0 \\ 0 & 0\end{array}\right) \cdot h^{-1}$ for some $g \in \mathrm{GL}_{p}(k), h \in \mathrm{GL}_{q}(k)$, where $s=\operatorname{rk} N$. As the tangent space to $\mathcal{V}_{p \times q}^{r}$ at $g \cdot N^{\prime} \cdot h^{-1}$ is $g \cdot \mathcal{T}_{\mathcal{v}_{p \times q}^{r}, N^{\prime}} \cdot h^{-1}$, it suffices to prove the claim for $N=\left(\begin{array}{cc}1_{s} & 0 \\ 0 & 0\end{array}\right)$.

It is obviously true for $s<r$. In case $s=r$, we decompose

$$
D=\left(\begin{array}{ll}
D_{11} & D_{12} \\
D_{21} & D_{22}
\end{array}\right)
$$


into blocks; the size of $D_{11}$ is $s \times s$. A straightforward computation yields that

$$
\mathcal{T}_{\mathcal{V}_{p \times q}^{r}, N}=\left\{D=\left(\begin{array}{ll}
D_{11} & D_{12} \\
D_{21} & D_{22}
\end{array}\right) ; D_{22}=0\right\} \text {. }
$$

But note that

$$
\operatorname{rk}\left(\begin{array}{cc}
N & D \\
0 & N
\end{array}\right)=\operatorname{rk}\left(\begin{array}{cccc}
1_{r} & 0 & D_{11} & D_{12} \\
0 & 0 & D_{21} & D_{22} \\
0 & 0 & 1_{r} & 0 \\
0 & 0 & 0 & 0
\end{array}\right)=2 r+\operatorname{rk} D_{22}
$$

So rk $\left(\begin{array}{cc}N & D \\ 0 & N\end{array}\right)=2 r$ if and only if $D_{22}=0$, and the lemma is established.

Let $\underline{a}$ be a $p \times q$-matrix with coefficients in $A$, set $L=\operatorname{Coker} v_{\underline{a}}$, and fix $N \in$ $e_{M, a}(k)$. Mapping the exact sequence

$$
A^{p} \stackrel{\nu_{\underline{a}}}{\longrightarrow} A^{q} \longrightarrow L \longrightarrow 0
$$

to a module $N^{\prime} \in \bmod _{A}^{d^{\prime}}(k)$ and identifying $\operatorname{Hom}_{A}\left(A, N^{\prime}\right)$ with $N^{\prime}$, we see that

$$
\operatorname{rk} N^{\prime}(\underline{a})+\operatorname{dim}_{k} \operatorname{Hom}_{A}\left(L, N^{\prime}\right)=q d^{\prime} .
$$

Corollary 7.4. Using the notions just introduced, we have that the tangent space $\mathcal{T}_{e_{M, L}, N}=\mathcal{T}_{e_{M, \underline{a}}, N}$ equals

(1) $\mathcal{T}_{\bmod _{A}^{d}, N}$ provided that $\operatorname{dim}_{k} \operatorname{Hom}_{A}(L, M)<\operatorname{dim}_{k} \operatorname{Hom}_{A}(L, N)$;

(2) $\left\{Z \in \mathcal{T}_{\bmod _{A}^{d}, N} ; \operatorname{dim}_{k} \operatorname{Hom}_{A}\left(L,\left(\begin{array}{cc}N & Z \\ 0 & N\end{array}\right)\right)=2 \operatorname{dim}_{k} \operatorname{Hom}_{A}(L, N)\right\}$ provided that $\operatorname{dim}_{k} \operatorname{Hom}_{A}(L, M)=\operatorname{dim}_{k} \operatorname{Hom}_{A}(L, N)$.

Proof. Remember that

$$
\varphi_{M, \underline{a}}=\Theta_{\underline{a}}^{-1}\left(\mathcal{V}_{p d \times q \underline{d}}^{\mathrm{rk} M(a)}\right) .
$$

Using (7.1), the corollary is a direct consequence of Lemma 7.3 and the fact that

$$
\operatorname{rk}\left(\begin{array}{cc}
N & Z \\
0 & N
\end{array}\right)(\underline{a})=\operatorname{rk}\left(\begin{array}{cc}
N(\underline{a}) & Z(\underline{a}) \\
0 & N(\underline{a})
\end{array}\right) .
$$

Now Proposition 7.1 is easy to prove. Indeed, we have

$$
\mathcal{T}_{e_{M}, N}=\bigcap \mathcal{T}_{e_{M, L}, N}
$$

where the intersection is taken over representatives $L$ of all isomorphism classes of $A$-modules which are finitely presented, or, by Lemma 3.4, even finite dimensional. In case $\operatorname{dim}_{k} \operatorname{Hom}_{A}(L, M)<\operatorname{dim}_{k} \operatorname{Hom}_{A}(L, N)$, this gives no restriction. The condition $\operatorname{dim}_{k} \operatorname{Hom}_{A}(L, M)=\operatorname{dim}_{k} \operatorname{Hom}_{A}(L, N)$ is equivalent to $L \in \operatorname{add} \mathcal{F}^{\prime}$ by definition, and having the equality

$$
\operatorname{dim}_{k} \operatorname{Hom}_{A}\left(L,\left(\begin{array}{cc}
N & Z \\
0 & N
\end{array}\right)\right)=2 \operatorname{dim}_{k} \operatorname{Hom}_{A}(L, N)
$$

for all $L \in \operatorname{add} \mathcal{F}^{\prime}$ is equivalent to $\varphi(Z) \in \mathcal{E}(N, N)$. 


\section{Singular loci}

In this last section, we assume $A$ to be representation finite, except for the final remark and example. All $A$-modules considered will be finite dimensional, and we fix $M \in \bmod _{A}^{d}(k), N \in \overline{\mathcal{O}}_{M}$. We denote the Auslander-Reiten quiver of $A$ by $\Gamma_{A}$. In order to study the singularity of $\boldsymbol{C}_{M}$ at $N$, we need some definitions and some preliminary results on source and sink maps, also called approximations by some authors.

We define the shadow $S$ of the degeneration from $M$ to $N$ to be the set of all meshes in $\Gamma_{A}$ which start in a vertex $X \notin \mathcal{F}$, or equivalently which stop in a vertex $X^{\prime} \notin \mathscr{F}^{\prime}$; the shadow $\delta_{\sigma}$ of an exact sequence $\sigma$ of $A$-modules consists of all meshes of $\Gamma_{A}$ with starting vertex $Y$ with $\delta_{\sigma}(Y)>0$ or equivalently with ending vertex $Y^{\prime}$ with $\delta_{\sigma}^{\prime}\left(Y^{\prime}\right)>0$. We call an exact sequence

$$
\sigma: 0 \longrightarrow Z \longrightarrow W \longrightarrow Y \longrightarrow 0
$$

fit for $(M, N)$ if its class $[\sigma]$ belongs to $\mathcal{E}(Y, Z)$, or equivalently if $\delta_{\sigma} \subseteq \delta$ or $\delta_{\sigma}(X)=0$ for all $X \in \mathcal{F}$.

For an $A$-module $Z$, we call a morphism $f: Z \rightarrow W$ a universal morphism from $Z$ to add $\mathscr{F}$ if $W \in \operatorname{add} \mathscr{F}$ and any morphism from $Z$ to some $W^{\prime} \in \operatorname{add} \mathcal{F}$ factors through $f$. It is easy to see that universal morphisms from $Z$ to add $\mathscr{F}$ exist. Such a morphism is necessarily injective as all injective indecomposables belong to $\mathcal{F}$. A universal morphism $f: Z \rightarrow W$ is called a source map if any endomorphism $\varphi$ of $W$ for which $\varphi \circ f$ is still universal is invertible. A source map $f_{Z}: Z \rightarrow W_{Z}$ is unique up to isomorphism, and it is characterized by the fact that the morphism $W_{Z} \rightarrow V_{Z}$ in the exact sequence

$$
\sigma_{Z}: 0 \longrightarrow Z \stackrel{f_{Z}}{\longrightarrow} W_{Z} \longrightarrow V_{Z} \longrightarrow 0
$$

is radical.

Sink maps from add $\mathcal{F}^{\prime}$ to some module $Y$ are defined dually. We will denote the exact sequence obtained from a sink map $W_{Y}^{\prime} \rightarrow Y$ from add $\mathscr{F}^{\prime}$ to $Y$ by

$$
\sigma_{Y}^{\prime}: 0 \longrightarrow U_{Y} \longrightarrow W_{Y}^{\prime} \longrightarrow Y \longrightarrow 0 .
$$

Lemma 8.1. Let $Y, Z$ be A-modules.

(1) The sequences $\sigma_{Z}$ and $\sigma_{Y}^{\prime}$ are fit for $(M, N)$.

(2) Mapping $Y$ to $\sigma_{Z}$, we obtain an exact sequence

$$
0 \rightarrow \operatorname{Hom}(Y, Z) \rightarrow \operatorname{Hom}\left(Y, W_{Z}\right) \rightarrow \operatorname{Hom}\left(Y, V_{Z}\right) \rightarrow \mathcal{E}(Y, Z) \rightarrow 0 .
$$

(3) Mapping $\sigma_{Y}^{\prime}$ to $Z$, we obtain an exact sequence

$$
0 \rightarrow \operatorname{Hom}(Y, Z) \rightarrow \operatorname{Hom}\left(W_{Y}^{\prime}, Z\right) \rightarrow \operatorname{Hom}\left(U_{Y}, Z\right) \rightarrow \mathcal{E}(Y, Z) \rightarrow 0 .
$$


(4) We have $S_{\sigma} \subseteq \delta_{\sigma_{Z}}$ and $S_{\sigma} \subseteq \delta_{\sigma_{Y}^{\prime}}$ for any exact sequence $\sigma$ with $[\sigma] \in \mathcal{E}(Y, Z)$.

Proof. Statement (1) holds by definition. For (2), note that the pullback of $\sigma_{Z}$ under any morphism in $\operatorname{Hom}\left(Y, V_{Z}\right)$ will still have a splitting pushout under any morphism from $Z$ to $X \in$ add $\mathcal{F}$ and thus belongs to $\mathcal{E}(Y, Z)$. By the definition of $\sigma_{Z}$, any exact sequence $\sigma$ with $[\sigma] \in \mathcal{E}(Y, Z)$ is a pullback of $\sigma_{Z}$. The proof of (3) is dual, and (4) follows from (2) and (3) as shadows cannot grow under pushouts nor under pullbacks.

As an immediate consequence we obtain the following corollary.

Corollary 8.2. $\operatorname{dim}_{k} \mathcal{E}(Y, Z)=\delta_{\sigma_{Y}^{\prime}}(Z)=\delta_{\sigma_{Z}}^{\prime}(Y)$.

Lemma 8.3. For an A-module $X$ the following properties are equivalent:

(1) $X \in \operatorname{add} \mathcal{F}$,

(2) $\mathcal{E}(-, X)=0$,

(3) $\varepsilon(N, X)=0$.

There is a dual statement characterizing $X^{\prime} \in \operatorname{add} \mathcal{F}^{\prime}$.

Proof. The implications from (1) to (2) and from (2) to (3) are immediate. In order to show that (3) implies (1), it is enough to prove the inclusion $S \subseteq \delta_{\sigma_{N}^{\prime}}$; in fact then both shadows coincide as $\sigma_{N}^{\prime}$ is fit for $(M, N)$. By [16] there is a short exact sequence

$$
\sigma: 0 \longrightarrow Z^{\prime} \longrightarrow Z^{\prime} \oplus M \longrightarrow N \longrightarrow 0 \text {. }
$$

By the definition of $\mathcal{F}$, we know that $\delta_{\sigma}=\delta$. Therefore $[\sigma] \in \mathcal{E}\left(N, Z^{\prime}\right)$, which implies that $S=S_{\sigma} \subseteq S_{\sigma_{N}^{\prime}} \subseteq S$ by Lemma 8.1 (4).

Lemma 8.4. The following conditions are equivalent:

(1) $U_{N}$ belongs to $\operatorname{add}\left(\mathcal{F}^{\prime}\right)$,

(2) $U_{Y}$ belongs to $\operatorname{add}\left(\mathcal{F}^{\prime}\right)$ for any module $Y$,

(3) $V_{N}$ belongs to $\operatorname{add}(\mathcal{F})$,

(4) $V_{Z}$ belongs to $\operatorname{add}(\mathcal{F})$ for any module $Z$.

Proof. Obviously (2) implies (1) and (4) implies (3). Thus, up to duality, it suffices to show that (1) implies (4). Let $Z$ be a module. As $W_{Z} \in \operatorname{add} \mathcal{F}$ and $W_{N}^{\prime} \in \operatorname{add} \mathcal{F}^{\prime}$, 
the exact sequences $\sigma_{N}^{\prime}$ and $\sigma_{Z}$ induce the following commutative diagram with exact rows and columns:

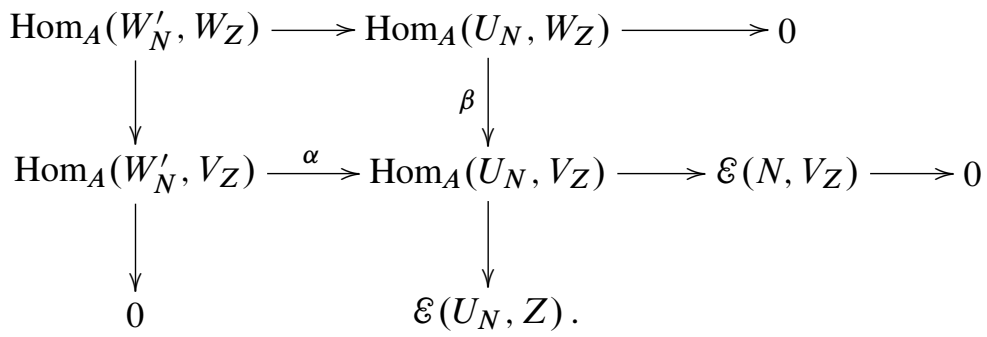

As $U_{N}$ belongs to add $\mathcal{F}^{\prime}$ by our hypothesis, $\beta$ is surjective. Then $\alpha$ is surjective as well, thus $\mathscr{E}\left(N, V_{Z}\right)=0$, which implies $V_{Z} \in$ add $\mathcal{F}$ by Lemma 8.3.

We are now ready to give a first characterization of the regularity $\ell_{M}$ at $N$.

Proposition 8.5. The scheme $\mathcal{C}_{M}$ is regular at $N$ if and only if $\mathcal{E}(M, M)=\{0\}$ and one of the equivalent conditions in Lemma 8.4 holds.

Proof. We compute the difference $\operatorname{dim}_{k} \mathscr{E}(N, N)-\operatorname{codim}(M, N)$. Observe that

$$
\operatorname{codim}(M, N)=\delta_{M, N}^{\prime}(N)+\delta_{M, N}(M) .
$$

By Corollary 8.2,

$$
\begin{aligned}
\operatorname{dim}_{k} \varepsilon(N, N)-\operatorname{dim}_{k} \mathcal{E}(N, M) & =\delta_{\sigma_{N}^{\prime}}(N)-\delta_{\sigma_{N}^{\prime}}(M) \\
& =\delta_{M, N}^{\prime}\left(U_{N} \oplus N\right)-\delta_{M, N}^{\prime}\left(W_{N}^{\prime}\right) \\
& =\delta_{M, N}^{\prime}\left(U_{N} \oplus N\right), \\
\operatorname{dim}_{k} \varepsilon(N, M)-\operatorname{dim}_{k} \mathcal{E}(M, M) & =\delta_{\sigma_{M}}^{\prime}(N)-\delta_{\sigma_{M}}^{\prime}(M) \\
& =\delta_{M, N}\left(M \oplus V_{M}\right)-\delta_{M, N}\left(W_{M}\right) \\
& =\delta_{M, N}\left(M \oplus V_{M}\right) .
\end{aligned}
$$

Thus

$$
\operatorname{dim}_{k} \varepsilon(N, N)-\operatorname{codim}(M, N)=\operatorname{dim}_{k} \varepsilon(M, M)+\delta_{M, N}^{\prime}\left(U_{N}\right)+\delta_{M, N}\left(V_{M}\right) .
$$

By Corollary 7.2, the scheme $\ell_{M}$ is regular at $N$ if and only if $\mathscr{E}(M, M)=0$ and $\delta_{M, N}^{\prime}\left(U_{N}\right)=\delta_{M, N}\left(V_{M}\right)=0$. As by Lemma $8.4 \delta_{M, N}^{\prime}\left(U_{N}\right)=0$ forces $\delta_{M, N}\left(V_{M}\right)=0$, our claim follows.

Lemma 8.6. Assume that $\mathscr{E}(M, M)=0$. Then $\boldsymbol{C}_{M}$ is singular at $N$ if and only if there exists an indecomposable $U$ such that the sequence

$$
\sigma_{U}: 0 \rightarrow U \stackrel{f_{U}}{\rightarrow} W_{U} \stackrel{g}{\rightarrow} V_{U} \rightarrow 0
$$

satisfies the following conditions: 
(1) The representation $V_{U}$ is indecomposable.

(2) The morphism $g: W_{U} \rightarrow V_{U}$ is a sink map from add $\mathcal{F}$ to $V_{U}$.

(3) If the mesh stopping at some indecomposable $Y$ belongs to $\mathcal{S}_{\sigma_{U}}$, then $Y \notin \mathcal{F}$.

Proof. We first prove that $\ell_{M}$ is singular at $N$ if these conditions hold. Note that $\sigma_{U}$ does not split, as $U \in \mathcal{F}$ would imply $V_{U}=0$, but $V_{U}$ is indecomposable. Therefore the mesh stopping at $V_{U}$ belongs to $\delta_{\sigma_{U}}$, and condition (3) implies $V_{U} \notin \mathscr{F}$. The claim then follows from Proposition 8.5 as condition (4) in Lemma 8.4 is violated.

In order to show the converse implication, observe that the surjection $W_{N} \rightarrow V_{N}$ factors through the sink map $\theta: C \rightarrow V_{N}$ from add $\mathcal{F}$ to $V_{N}$. In particular $\theta$ is surjective and we obtain the following commutative diagram with exact rows:

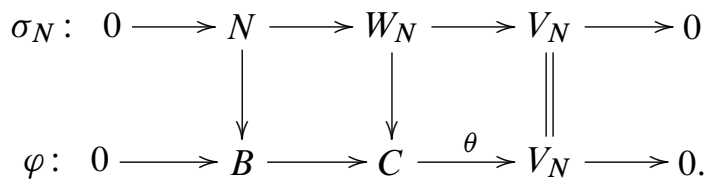

Thus $\varphi$ is fit for $(M, N)$, being a pushout of $\sigma_{N}$. A decomposition of $V_{N}$ into a direct sum of submodules yields a corresponding decomposition of $\varphi$ as a direct sum. We choose a direct summand of $\varphi$ :

$$
\eta: 0 \longrightarrow U \stackrel{f}{\longrightarrow} W \stackrel{g}{\longrightarrow} V \longrightarrow 0
$$

such that $V$ is indecomposable and does not belong to $\operatorname{add}(\mathcal{F})$. As $g$ is radical, $f$ is a source map from $U$ to add $\mathcal{F}$. But a source map from a decomposable module has a decomposable cokernel, and therefore $U$ must be indecomposable and $\eta$ is isomorphic to $\sigma_{U}$.

Finally, suppose the mesh stopping at some indecomposable $Y$ belongs to $S_{\eta}$. Equivalently, we have $\delta_{\eta}^{\prime}(Y) \neq 0$. If $Y$ belongs to $\mathcal{F}$, any morphism from $Y$ to $V$ factors through $g$, and thus $\delta_{\eta}^{\prime}(Y)=0$, because $g$ is a sink map from add $\mathcal{F}$ to $V$. Our last claim follows.

Lemma 8.7. Assume that the algebra $A$ is directed and consider the exact sequence $\eta$ from Lemma 8.6. Then $\operatorname{codim}(W, U \oplus V)=1$.

Proof. Since $\eta$ is fit for $(M, N)$ and $W$ belongs to $\operatorname{add}(\mathcal{F})$, we have $\delta_{\eta}(W)=0$. Since $U$ and $V$ are indecomposable and $A$ is directed, $\operatorname{Ext}_{A}^{1}(U, U)=\{0\}$ and $\operatorname{End}_{A}(V) \simeq k$. We conclude from the long exact sequences

$$
\begin{aligned}
& 0 \rightarrow \operatorname{Hom}_{A}(U, U) \rightarrow \operatorname{Hom}_{A}(U, W) \rightarrow \operatorname{Hom}_{A}(U, V) \rightarrow \operatorname{Ext}_{A}^{1}(U, U), \\
& 0 \rightarrow \operatorname{Hom}_{A}(V, U) \rightarrow \operatorname{Hom}_{A}(V, W) \rightarrow \operatorname{Hom}_{A}(V, V) \rightarrow \mathcal{E}(V, U) \rightarrow 0
\end{aligned}
$$

induced by $\eta$ that $\delta_{\eta}^{\prime}(U)=0$ and $\delta_{\eta}^{\prime}(V)=1$. Thus

$$
\operatorname{codim}(W, U \oplus V)=\delta_{\eta}^{\prime}(U \oplus V)+\delta_{\eta}(W)=1 .
$$


We end this section with a few remarks on singularities.

Remark 8.8. For a finitely generated algebra $A$ and $M \in \bmod _{A}^{d}(k)$, we have a chain of inclusions of schemes $\overline{\mathcal{O}}_{M} \subseteq\left(\mathcal{C}_{M}\right)_{\text {red }} \subseteq \mathcal{C}_{M}$. Fix $N \in \overline{\mathcal{O}}_{M}$, and let us compare regularity at $N$ for $\overline{\mathcal{O}}_{M},\left(\varphi_{M}\right)_{\text {red }}$, and $\ell_{M}$. Clearly, if $\ell_{M}$ is regular at $N$, the subscheme $\left(\mathcal{C}_{M}\right)_{\text {red }}$ will be as well. Remember that in Example 3.7 we have that $\left(\mathcal{C}_{M}\right)_{\text {red }}=\overline{\mathcal{O}}_{M}$ and that the tangent space of $\overline{\mathcal{O}}_{M}$ (and thus of $\left.\left(\mathcal{C}_{M}\right)_{\text {red }}\right)$ at $N$ is a proper subspace of $\mathcal{T}_{\mathscr{C}_{M}, N}$. So there might be cases where $\mathscr{C}_{M}$ is singular at $N$ while $\left(\mathcal{C}_{M}\right)_{\text {red }}$ is regular at that point.

As $\overline{\mathcal{O}}_{M}$ is an irreducible component of $\left(\mathcal{C}_{M}\right)_{\text {red }}$ by Proposition 1 of [4], we have that regularity of $\left(\mathcal{C}_{M}\right)_{\text {red }}$ at $N$ implies regularity of $\overline{\mathcal{O}}_{M}$ at $N$. The following simplified version of Carlson's example shows that the reverse implication is false.

Example 8.9. Let $Q$ be the quiver

$$
1 \stackrel{\alpha}{\stackrel{2}{\longrightarrow}} 2 \underset{\delta}{\stackrel{\gamma}{\longrightarrow}} 3
$$

and let $J$ be the ideal generated by $\delta \alpha, \gamma \beta$ and $\gamma \alpha-\delta \beta$. Consider the representations

$$
M=k \stackrel{\left(\begin{array}{l}
1 \\
0
\end{array}\right)}{\underset{\left(\begin{array}{l}
0 \\
1
\end{array}\right)}{\longrightarrow}} k^{2} \underset{\left(\begin{array}{ll}
1 & 0
\end{array}\right)}{\underset{\left(\begin{array}{ll}
0 & 1
\end{array}\right)}{\longrightarrow}} k, \quad U_{\lambda}=0 \underset{(0)}{\stackrel{(0)}{\longrightarrow}} k \underset{(\lambda)}{\stackrel{(1)}{\longrightarrow}} k,
$$

and

$$
V_{\mu}=k \underset{(1)}{\stackrel{(\mu)}{\longrightarrow}} k \stackrel{(0)}{\underset{(0)}{\longrightarrow}} 0,
$$

for $\lambda, \mu \in k$. It is not difficult to see and can be found in [14] that $\overline{\mathcal{O}}_{M}$ and the closure of $\bigcup_{\lambda, \mu \in k} \mathrm{GL}_{\boldsymbol{d}}(k) *\left(U_{\lambda} \oplus V_{\mu}\right)$ are irreducible components of $\boldsymbol{C}_{\boldsymbol{M}}$ and that they intersect in the closure of $\bigcup_{\lambda \in k} \operatorname{GL}_{\boldsymbol{d}}(k) *\left(U_{\lambda} \oplus V_{-\lambda}\right)$, where $\boldsymbol{d}=(1,2,1)$. So $\ell_{M}$ is singular at $N=U_{1} \oplus V_{-1}$.

On the other hand, a computation shows that the morphism given by

$$
k \stackrel{\left(\begin{array}{l}
x_{1} \\
x_{2}
\end{array}\right)}{\stackrel{\left(\begin{array}{ll}
y_{1} \\
y_{2}
\end{array}\right)}{\longrightarrow}} k^{2} \underset{\left(\begin{array}{ll}
t_{1} & z_{2}
\end{array}\right)}{\longrightarrow} k \mapsto\left(\begin{array}{cccc}
t_{1} & x_{2} & y_{1} & y_{2} \\
-t_{2} & t_{1} & z_{2} & -z_{1}
\end{array}\right)
$$


is an isomorphism from $\overline{\mathcal{O}}_{M}$ to the variety $\mathcal{V}_{2 \times 4}^{1}(k)$ of $2 \times 4$-matrices of rank at most 1 , which has a single singularity at 0 , the image of the semisimple representation. Hence $\overline{\mathcal{O}}_{M}$ is regular at $N$.

\section{References}

[1] G. Bobiński and G. Zwara, Normality of orbit closures for Dynkin quivers of type $\mathbb{A}_{n}$. Manuscripta Math. 105 (2001), 103-109. Zbl 1031.16012 MR 1885816

[2] G. Bobiński and G. Zwara, Schubert varieties and representations of Dynkin quivers. Colloq. Math. 94 (2002), 285-309. Zbl 1013.14011 MR 1967381

[3] K. Bongartz, A geometric version of the Morita equivalence. J. Algebra 139 (1991), 159-171. Zbl 0787.16011 MR 1106345

[4] K. Bongartz, Degenerations for representations of tame quivers. Ann. Sci. École Norm. Sup. 28 (1995), 647-668. Zbl 0844.16007 MR 1341664

[5] K. Bongartz, On degenerations and extensions of finite dimensional modules. Adv. Math. 121 (1996), 245-287. Zbl 0862.16007 MR 1402728

[6] K. Bongartz, Some geometric aspects of representation theory. In Algebras and modules. I. CMS Conf. Proc. 23, Amer. Math. Soc., Providence, RI, 1998, 1-27. Zbl 0915.16008 MR 1648601

[7] W. Bruns and U. Vetter, Determinantal rings. Lecture Notes in Math. 1327, SpringerVerlag, Berlin 1988. Zbl 0673.13006 MR 0953963

[8] D. Eisenbud, Commutative algebra. With a view toward algebraic geometry. Grad. Texts in Math. 150, Springer-Verlag, New York 1995. Zbl 0819.13001 MR 1322960

[9] P. Gabriel, Finite representation type is open. In Representations of algebras, Lecture Notes in Math. 488, Springer-Verlag, Berlin 1975, 132-155. Zbl 0313.16034 MR 0376769

[10] A. Grothendieck and J. A. Dieudonné, Éléments de géométrie algébrique IV. Inst. Hautes Études Sci. Publ. Math. 32 (1967). Zbl 0153.22301 MR 0238860

[11] W. Hesselink, Singularities in the nilpotent scheme of a classical group. Trans. Amer. Math. Soc. 222 (1976), 1-32. Zbl 0332.14017 MR 0429875

[12] V. Lakshmibai and P. Magyar, Degeneracy schemes, quiver schemes, and Schubert varieties. Internat. Math. Res. Notices 12 (1998), 627-640. Zbl 0936.14001 MR 1635873

[13] Ch. Riedtmann, Degenerations for representations of quivers with relations. Ann. Sci. École Norm. Sup. 4 (1986), 275-301. Zbl 0603.16025 MR 0868301

[14] Ch. Riedtmann, Geometry of modules: degenerations. In Trends in the representation theory of finite-dimensional algebras, Contemp. Math. 229, Amer. Math. Soc., Providence, RI, 1998, 281-291. Zbl 0927.16007 MR 1676226

[15] G. Zwara, Degenerations for modules over representation-finite algebras. Proc. Amer. Math. Soc. 127 (1999), 1313-1322. Zbl 0927.16008 MR 1476404

[16] G. Zwara, Degenerations of finite dimensional modules are given by extensions. Compos. Math. 121 (2000), 205-218. Zbl 0957.16007 MR 1757882

[17] G. Zwara, Smooth morphisms of module schemes. Proc. London Math. Soc. 84 (2002), 539-558. Zbl 1054.16009 MR 1888422 
Received December 7, 2009

Christine Riedtmann, Mathematisches Institut, Universität Bern, Sidlerstrasse 5, 3012 Bern, Switzerland

E-mail: christine.riedtmann@math.unibe.ch

Grzegorz Zwara, Faculty of Mathematics and Computer Science, Nicolaus Copernicus University, Chopina 12/18, 87-100 Toruń, Poland

E-mail: gzwara@mat.umk.pl 